\title{
LOWER LARGE DEVIATIONS FOR THE MAXIMAL FLOW THROUGH TILTED CYLINDERS IN TWO-DIMENSIONAL FIRST PASSAGE PERCOLATION
}

\author{
RAPhaËL Rossignol ${ }^{1}$ And MARIE ThÉRET ${ }^{2}$
}

\begin{abstract}
Equip the edges of the lattice $\mathbb{Z}^{2}$ with i.i.d. random capacities. A law of large numbers is known for the maximal flow crossing a rectangle in $\mathbb{R}^{2}$ when the side lengths of the rectangle go to infinity. We prove that the lower large deviations are of surface order, and we prove the corresponding large deviation principle from below. This extends and improves previous large deviations results of Grimmett and Kesten [9] obtained for boxes of particular orientation.
\end{abstract}

Mathematics Subject Classification. 60K35, 82B43.

Received July 3, 2009.

\section{INTRODUCTION}

Imagine each edge of $\mathbb{Z}^{2}$ is a microscopic pipe some fluid can go through. To each edge $e$, we attach a random capacity $t(e)$ and suppose that all these random variables are independent and identically distributed with common distribution function $F$ on $\mathbb{R}^{+}$. Now, we take a large rectangle $R$ in $\mathbb{R}^{2}$, decide that one side is the "left side", and accordingly name the other sides the right side, the top and the bottom of $R$. We are concerned with the maximal flow rate that can cross $R$ from the top to the bottom, while never exceeding the capacities of the edges (see Sect. 2.2 for a formal definition). Informally, we ask: how does the maximal flow between the top and the bottom behave when $R$ gets larger and larger? This question was first considered in Grimmett and Kesten [9], where a law of large numbers and large deviation estimates where proved, but only for "straight" rectangles $R$, i.e. with sides parallel to the coordinate axes. Let us mention that lower large deviations are of surface order, i.e. of the order of the width of $R$ whereas upper large deviations are of volume order, i.e. of the order of the area of $R$. In a previous work Rossignol and Théret [13], the authors extended the law of large numbers of Grimmett and Kesten [9] to rectangles $R$ with arbitrary orientation. The purpose of this article is to give a corresponding lower large deviation principle.

We shall precise now our contribution. Let us notice that the problem of maximal flow has been studied in the more general case of the lattice $\mathbb{Z}^{d}, R$ being then some box in $\mathbb{R}^{d}$, first through the work of Chayes and Chayes [7] and Kesten [12], and then notably with Zhang [19,20], Théret [15,16], Rossignol and Théret [14].

\footnotetext{
Keywords and phrases. First passage percolation, maximal flow, large deviation principle.

1 Université Paris Sud, Laboratoire de Mathématiques, bâtiment 425, 91405 Orsay Cedex, France.

raphael.rossignol@math.u-psud.fr

2 École Normale Supérieure, Département de Mathématiques et Applications, 45 rue d'Ulm, 75230 Paris Cedex 05, France. marie.theret@ens.fr
} 
In any case, it is much simpler to study a subadditive version of the maximal flow, which we shall call $\tau$ in Section 2.2 below. Then, when $R$ is straight (i.e. has faces parallel to coordinate hyperplanes), one can pass from $\tau$ to the maximal flow thanks to symmetry considerations. When $d \geq 3$, results concerning $R$ such as large deviations estimates or laws of large numbers and that do not suppose that $R$ is straight (or even that it is a box) are known from the recent works Cerf and Théret [4-6], but they require a lot of geometric work, need strong moment hypotheses and above all have not yet provided large deviation principles. Moreover they are in any case much more involved than what is expected to be necessary in two dimensions to treat the simple case of rectangles. Indeed, in two dimensions, duality considerations are of great help to prove a law of large numbers and a lower large deviation principle. The aim of Rossignol and Théret [13] was thus to derive in a simple way the law of large numbers for the maximal flow from the top to the bottom through a rectangle $R$. The aim of the present article is to use the same constructions to derive in a simple way the lower large deviation principle of the maximal flow that is still missing in the works of Cerf and Théret (see [5]), and to provide a deep study of the properties of the corresponding rate function. A similar, simple proof in dimension $d \geq 3$ remains to be found.

The precise definitions and notations, the relevant background as well as the main results are presented in Section 2. Then, the main construction and the lower large deviation estimates are the purpose of Section 3, while Section 4 contains the proof of the large deviation principle itself and the study of the rate function.

\section{Notations, BACKGRound AND MAin REsults}

The most important notations are gathered in Sections 2.1 to 2.3, the relevant background is described in Section 2.4 while our main results are stated in Section 2.5. We discuss in section 2.6 the different hypotheses appearing in our results.

\subsection{Maximal flow on a graph}

First, let us define the notion of a flow on a finite unoriented graph $G=(V, \mathcal{E})$ with set of vertices $V$ and set of edges $\mathcal{E}$. Let $t=(t(e))_{e \in \mathcal{E}}$ be a collection of non-negative real numbers, which are called capacities. It means that $t(e)$ is the maximal amount of fluid that can go through the edge $e$ per unit of time. To each edge $e$, one may associate two oriented edges, and we shall denote by $\overrightarrow{\mathcal{E}}$ the set of all these oriented edges. Let $A$ and $Z$ be two finite, disjoint, non-empty sets of vertices of $G$ : $A$ denotes the source of the network, and $Z$ the sink. A function $\theta$ on $\overrightarrow{\mathcal{E}}$ is called a flow from $A$ to $Z$ with strength $\|\theta\|$ and capacities $t$ if it is antisymmetric, i.e. $\theta_{\overrightarrow{x y}}=-\theta_{\overrightarrow{y x}}$, if it satisfies the node law at each vertex $x$ of $V \backslash(A \cup Z)$ :

$$
\sum_{y \sim x} \theta_{\overrightarrow{x y}}=0
$$

where $y \sim x$ means that $y$ and $x$ are neighbours on $G$, if it satisfies the capacity constraints:

$$
\forall e \in \mathcal{E},|\theta(e)| \leq t(e)
$$

and if the "flow in" at $A$ and the "flow out" at $Z$ equal $\|\theta\|$ :

$$
\|\theta\|=\sum_{a \in A} \sum_{\substack{y \sim a \\ y \notin A}} \theta(\overrightarrow{a y})=\sum_{z \in Z} \sum_{\substack{y \sim z \\ y \notin Z}} \theta(\overrightarrow{y z}) .
$$

The maximal flow from $A$ to $Z$, denoted by $\phi_{t}(G, A, Z)$, is defined as the maximum strength of all flows from $A$ to $Z$ with capacities $t$. We shall in general omit the subscript $t$ when it is understood from the context. The max-flow min-cut theorem (see Bollobás [2] for instance) asserts that the maximal flow from $A$ to $Z$ equals the minimal capacity of a cut between $A$ and $Z$. Precisely, let us say that $E \subset \mathcal{E}$ is a cut between $A$ and $Z$ in $G$ 
if every path from $A$ to $Z$ borrows at least one edge of $E$. Define $V(E)=\sum_{e \in E} t(e)$ to be the capacity of a cut $E$. Then,

$$
\phi_{t}(G, A, Z)=\min \{V(E) \text { s.t. } E \text { is a cut between } A \text { and } Z \text { in } G\} \text {. }
$$

\subsection{On the square lattice}

We shall always consider $G$ as a piece of $\mathbb{Z}^{2}$. More precisely, we consider the graph $\mathbb{L}=\left(\mathbb{Z}^{2}, \mathbb{E}^{2}\right)$ having for vertices $\mathbb{Z}^{2}$ and for edges $\mathbb{E}^{2}$, the set of pairs of nearest neighbours for the standard $L^{1}$ norm. The notation $\langle x, y\rangle$ corresponds to the edge with endpoints $x$ and $y$. To each edge $e$ in $\mathbb{E}^{2}$ we associate a random variable $t(e)$ with values in $\mathbb{R}^{+}$. We suppose that the family $\left(t(e), e \in \mathbb{E}^{2}\right)$ is independent and identically distributed, with a common distribution function $F$. More formally, we take the product measure $\mathbb{P}=F^{\otimes \Omega}$ on $\Omega=\prod_{e \in \mathbb{E}^{2}}[0, \infty[$, and we write its expectation $\mathbb{E}$. If $G$ is a subgraph of $\mathbb{L}$, and $A$ and $Z$ are two subsets of vertices of $G$, we shall denote by $\phi(G, A, Z)$ the maximal flow in $G$ from $A$ to $Z$, where $G$ is equipped with capacities $t$. When $B$ is a subset of $\mathbb{R}^{2}$, and $A$ and $Z$ are subsets of $\mathbb{Z}^{2} \cap B$, we shall denote by $\phi(B, A, Z)$ again the maximal flow $\phi(G, A, Z)$ where $G$ is the induced subgraph of $\mathbb{Z}^{2}$ with set of vertices $\mathbb{Z}^{2} \cap B$.

For all $\theta \in \mathbb{R}$, we define the two following vectors, of unit Euclidean nom,

$$
\vec{v}(\theta)=(\cos \theta, \sin \theta) \quad \text { and } \quad \vec{v}^{\perp}(\theta)=(\sin \theta,-\cos \theta)
$$

We denote by $\vec{e}_{1}$ (resp. $\left.\vec{e}_{2}\right)$ the vector $(1,0) \in \mathbb{R}^{2}$ (resp. $(0,1)$ ). Let $A$ be a non-empty line segment in $\mathbb{R}^{2}$. We shall denote by $l(A)$ its (Euclidean) length. All line segments will be supposed to be closed in $\mathbb{R}^{2}$. There exists $\theta \in[0, \pi[$ such that $\vec{v}(\theta)$ is orthogonal to hyp $(A)$, the hyperplane spanned by $A$. Denote by $a$ and $b$ the end-points of $A$ such that $(b-a) \cdot \vec{v}^{\perp}(\theta)>0$. For $h$ a positive real number, we denote by cyl $(A, h)$ the cylinder of basis $A$ and height $2 h$, i.e., the set

$$
\operatorname{cyl}(A, h)=\{x+t \vec{v}(\theta) \mid x \in A, t \in[-h, h]\} .
$$

We define also the r-neighbourhood $\mathcal{V}(H, r)$ of a subset $H$ of $\mathbb{R}^{d}$ as

$$
\mathcal{V}(H, r)=\left\{x \in \mathbb{R}^{2} \mid d(x, H)<r\right\},
$$

where the distance is the Euclidean one $\left(d(x, H)=\inf \left\{\|x-y\|_{2} \mid y \in H\right\}\right)$.

Now, we define $D(A, h)$ the set of admissible boundary conditions on cyl $(A, h)$ (see Fig. 1):

$$
D(A, h)=\left\{(k, \tilde{\theta}) \mid k \in[0,1] \text { and } \tilde{\theta} \in\left[\theta-\arctan \left(\frac{2 h k}{l(A)}\right), \theta+\arctan \left(\frac{2 h(1-k)}{l(A)}\right)\right]\right\} .
$$

The meaning of an element $\kappa=(k, \tilde{\theta})$ of $D(A, h)$ is the following. In $\operatorname{cyl}(A, h)$, we may define two points $c$ and $d$ such that $c$ is "at height $2 k h$ on the left side of $\operatorname{cyl}(A, h)$ ", and $d$ is "on the right $\operatorname{side}$ of $\operatorname{cyl}(A, h)$ " by

$$
c=a+(2 k-1) h \vec{v}(\theta), \quad(d-c) \text { is orthogonal to } \vec{v}(\widetilde{\theta}) \quad \text { and } d \text { satisfies } \overrightarrow{c d} \cdot \vec{v}^{\perp}(\widetilde{\theta})>0
$$

Then we see that $D(A, h)$ is exactly the set of parameters so that $c$ and $d$ remain "on the $\operatorname{sides}$ of $\operatorname{cyl}(A, h)$ ".

We define also $\mathcal{D}(A, h)$, the set of angles $\tilde{\theta}$ such that there is an admissible boundary condition with angle $\tilde{\theta}$ :

$$
\mathcal{D}(A, h)=\left[\theta-\arctan \left(\frac{2 h}{l(A)}\right), \theta+\arctan \left(\frac{2 h}{l(A)}\right)\right] .
$$

It will be useful to define the left side (resp. right side) of $\operatorname{cyl}(A, h)$ : let $\operatorname{left}(A)(\operatorname{resp} . \operatorname{right}(A))$ be the set of vertices in $\operatorname{cyl}(A, h) \cap \mathbb{Z}^{2}$ such that there exists $y \notin \operatorname{cyl}(A, h),\langle x, y\rangle \in \mathbb{E}^{d}$ and $[x, y[$, the segment that includes $x$ and excludes $y$, intersects $a+[-h, h] . \vec{v}(\theta)$ (resp. $b+[-h, h] . \vec{v}(\theta))$. 


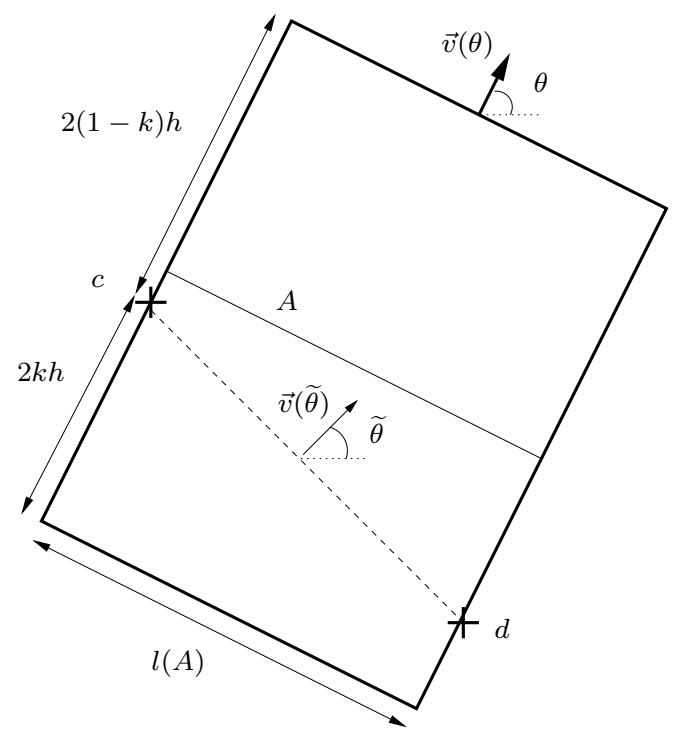

Figure 1. An admissible boundary condition $(k, \widetilde{\theta})$.

Now, the set $\operatorname{cyl}(A, h) \backslash(c+\mathbb{R}(d-c))$ has two connected components, which we denote by $\mathcal{C}_{1}(A, h, k, \tilde{\theta})$ and $\mathcal{C}_{2}(A, h, k, \tilde{\theta})$. For $i=1,2$, let $A_{i}^{h, k, \tilde{\theta}}$ be the set of the points in $\mathcal{C}_{i}(A, h, k, \tilde{\theta}) \cap \mathbb{Z}^{2}$ which have a nearest neighbour in $\mathbb{Z}^{2} \backslash \operatorname{cyl}(A, h)$ :

$$
A_{i}^{h, k, \tilde{\theta}}=\left\{x \in \mathcal{C}_{i}(A, h, k, \tilde{\theta}) \cap \mathbb{Z}^{2} \mid \exists y \in \mathbb{Z}^{2} \backslash \operatorname{cyl}(A, h),\|x-y\|_{1}=1\right\} .
$$

We define the flow in $\operatorname{cyl}(A, h)$ constrained by the boundary condition $\kappa=(k, \tilde{\theta})$ as:

$$
\phi^{\kappa}(A, h):=\phi\left(\operatorname{cyl}(A, h), A_{1}^{h, k, \tilde{\theta}}, A_{2}^{h, k, \tilde{\theta}}\right) .
$$

A special role is played by the condition $\kappa=(1 / 2, \theta)$, and we shall denote:

$$
\tau(A, h)=\tau(\operatorname{cyl}(A, h), \vec{v}(\theta)):=\phi^{(1 / 2, \theta)}(A, h) .
$$

Let $T(A, h)$ (respectively $B(A, h))$ be the top (respectively the bottom) of $\operatorname{cyl}(A, h)$, i.e.,

$$
T(A, h)=\left\{x \in \operatorname{cyl}(A, h) \mid \exists y \notin \operatorname{cyl}(A, h),\langle x, y\rangle \in \mathbb{E}^{d} \text { and }\langle x, y\rangle \text { intersects } A+h \vec{v}(\theta)\right\}
$$

and

$$
B(A, h)=\left\{x \in \operatorname{cyl}(A, h) \mid \exists y \notin \operatorname{cyl}(A, h),\langle x, y\rangle \in \mathbb{E}^{d} \text { and }\langle x, y\rangle \text { intersects } A-h \vec{v}(\theta)\right\} .
$$

We shall denote the flow in $\operatorname{cyl}(A, h)$ from the top to the bottom as:

$$
\phi(A, h)=\phi(\operatorname{cyl}(A, h), \vec{v}(\theta))=\phi(\operatorname{cyl}(A, h), T(A, h), B(A, h)) .
$$

\subsection{Duality}

The main reason why dimension 2 is easier to deal with than dimension $d \geq 3$ is duality. Indeed in dimension 2 , cuts between the top and the bottom of a cylinder are in 1 to 1 correspondance with paths from left to right in the dual lattice. This allows to relate unconstrained flows to constrained flows, as shows the following lemma taken from Rossignol and Théret [13]. 
Lemma 2.1. Let $A$ be any line segment in $\mathbb{R}^{2}$ and $h$ a positive real number. Then,

$$
\phi(A, h)=\min _{\kappa \in D(A, h)} \phi^{\kappa}(A, h)
$$

Notice that the condition $\kappa$ belongs to the non-countable set $D(A, h)$, but the graph is discrete so $\phi^{\kappa}(A, h)$ takes only a finite number of values when $\kappa \in D(A, h)$. Precisely, there is a finite subset $\tilde{D}(A, h)$ of $D(A, h)$, such that:

$$
\operatorname{card}(\tilde{D}(A, h)) \leq C_{0} h^{2}
$$

for some universal constant $C_{0}$, and:

$$
\phi(A, h)=\min _{\kappa \in \tilde{D}(A, h)} \phi^{\kappa}(A, h) .
$$

\subsection{Background}

We gather in this section known results concerning the behaviour of the variables $\tau(n A, h(n))$ and $\phi(n A, h(n))$ when $n$ and $h(n)$ go to infinity. They are of two types. We present first the law of large numbers satisfied by both variables. Then we describe the large deviation principle from below proved for $\tau(n A, h(n))$. The purpose of this article is to extend the study of lower large deviations to the variable $\phi(n A, h(n))$.

\begin{tabular}{|c|c|}
\hline Hypotheses on $F$ & Hypotheses on $h$ \\
\hline $\begin{array}{ll}\text { (F1) } & F(0)<1-p_{c}(2)=\frac{1}{2} \\
\text { (F2) } & \int_{0}^{\infty} x d F(x)<\infty \\
\text { (F3) } & \int_{0}^{\infty} x^{2} d F(x)<\infty \\
\text { (F4) } & \exists \gamma>0, \int_{0}^{\infty} \mathrm{e}^{\gamma x} d F(x)<\infty \\
\text { (F5) } & \forall \gamma>0, \int_{0}^{\infty} \mathrm{e}^{\gamma x} d F(x)<\infty\end{array}$ & $\begin{array}{ll}\text { (H1) } & \lim _{n \rightarrow \infty} h(n)=+\infty \\
\text { (H2) } & \lim _{n \rightarrow \infty} \frac{\log h(n)}{n}=0 \\
\text { (H3) } & \lim _{n \rightarrow \infty} \frac{h(n)}{n}=+\infty \\
\text { (H4) } & \exists \alpha \in\left[0, \frac{\pi}{2}\right], \lim _{n \rightarrow \infty} \frac{2 h(n)}{n l(A)}=\tan \alpha\end{array}$ \\
\hline
\end{tabular}

We gather the main hypotheses that we shall do on $F$ and on the height $h$. Notice that $(\mathbf{F 5}) \Rightarrow(\mathbf{F 4}) \Rightarrow$ $(\mathbf{F 3}) \Rightarrow(\mathbf{F} 2)$ and $(\mathbf{H 3}) \Rightarrow(\mathbf{H 1})$.

Using a subadditive argument and deviation inequalities, Rossignol and Théret have proved in Rossignol and Théret [14] that $\tau(n A, h(n))$ satisfies a law of large numbers:

Theorem 2.2. We suppose that (F2) holds. For every unit vector $\vec{v}(\theta)=(\cos \theta, \sin \theta)$, there exists a constant $\nu_{\theta}$ depending on $F$ and $\theta$, such that for every non-empty line-segment $A$ orthogonal to $\vec{v}(\theta)$ and of Euclidean length $l(A)$, for every height function $h: \mathbb{N} \rightarrow \mathbb{R}^{+}$satisfying $(\mathbf{H 1})$, we have

$$
\lim _{n \rightarrow \infty} \frac{\tau(n A, h(n))}{n l(A)}=\nu_{\theta} \quad \text { in } L^{1} .
$$

Moreover, if the origin of the graph belongs to A, or if (F3) holds, then

$$
\lim _{n \rightarrow \infty} \frac{\tau(n A, h(n))}{n l(A)}=\nu_{\theta} \quad \text { a.s. }
$$

This law of large numbers holds in fact for every dimension $d \geq 2$, and the limit $\nu$ depends also on the dimension. Let us remark that (in dimension two) $\nu_{\theta}$ is equal to $\mu\left(\vec{v}^{\perp}(\theta)\right.$ ), where $\mu($.$) is the time-constant$ function of first passage percolation as defined in Kesten [11], (3.10) p. 158. This equality follows from the duality considerations of Section 2.3 and standard first passage percolation techniques (see also Thm. 5.1 in Grimmett and Kesten [9]) that relate point-to-point passage times in cylinders to unrestricted point-to-point passage times (as in Hammersley and Welsh [10], Thm. 4.3.7 for instance). Boivin has also proved a very similar 
law of large numbers (see Thm. 6.1 in Boivin [1]). Notice that for the definition of $\mu($.$) , Kesten requires only$ the existence of the first moment of the law $F$ in the proof from Kesten [11], and it can also be defined under the weaker condition $\int_{0}^{\infty}(1-F(x))^{4} \mathrm{~d} x<\infty$. In fact, a time constant can even be defined without any moment condition at the price of changing slightly the definition of first passage time (see Kesten [11]).

One consequence of this equality between $\nu$ and $\mu$ is that $\theta \mapsto \nu_{\theta}$ is either constant equal to zero, or always non-zero. In fact the following property holds ( $c f$. Kesten [11], Thm. 6.1 and Rem. 6.2 p. 218):

Proposition 2.3. We suppose that (F2) holds. Then $\nu_{\theta}$ is well defined for all $\theta$, and we have

$$
\exists \theta \text { s.t. } \nu_{\theta}>0 \Longleftrightarrow \forall \theta \nu_{\theta}>0 \Longleftrightarrow(\mathbf{F 1}) .
$$

We recall that for all $n \in \mathbb{N}$, we have defined

$$
\mathcal{D}(n A, h(n))=\left[\theta-\arctan \left(\frac{2 h(n)}{n l(A)}\right), \theta+\arctan \left(\frac{2 h(n)}{n l(A)}\right)\right] .
$$

Extending the law of large numbers proved by Grimmett and Kesten [9] for $\phi(n A, h(n))$ in boxes of particular orientation, the authors proved the following result (see Thm. 2.8 and Cor. 2.10 in Rossignol and Théret [13]), in the same spirit as the result of Garet [8]:

Theorem 2.4. Let $A$ be a non-empty line-segment in $\mathbb{R}^{2}$, with Euclidean length $l(A)$. Let $\theta \in[0, \pi[$ be such that $(\cos \theta, \sin \theta)$ is orthogonal to $A$ and $h: \mathbb{N} \rightarrow \mathbb{R}^{+}$satisfying $(\mathbf{H 1})$ and $(\mathbf{H 2})$. Define:

$$
\overline{\mathcal{D}}=\limsup _{n \rightarrow \infty} \mathcal{D}(n A, h(n))=\bigcap_{N \geq 1} \bigcup_{n \geq N} \mathcal{D}(n A, h(n)),
$$

and

$$
\underline{\mathcal{D}}=\liminf _{n \rightarrow \infty} \mathcal{D}(n A, h(n))=\bigcup_{N \geq 1} \bigcap_{n \geq N} \mathcal{D}(n A, h(n)) .
$$

Suppose that (F2) holds. Then,

$$
\liminf _{n \rightarrow \infty} \frac{\mathbb{E}[\phi(n A, h(n))]}{n l(A)}=\inf \left\{\frac{\nu_{\widetilde{\theta}}}{\cos (\widetilde{\theta}-\theta)} \mid \widetilde{\theta} \in \overline{\mathcal{D}}\right\}
$$

and

$$
\limsup _{n \rightarrow \infty} \frac{\mathbb{E}[\phi(n A, h(n))]}{n l(A)}=\inf \left\{\frac{\nu_{\tilde{\theta}}}{\cos (\widetilde{\theta}-\theta)} \mid \widetilde{\theta} \in \underline{\mathcal{D}}\right\} .
$$

Moreover, if the origin of the graph 0 is the middle of $A$, or if (F3) holds, then

$$
\liminf _{n \rightarrow \infty} \frac{\phi(n A, h(n))}{n l(A)}=\inf \left\{\frac{\nu_{\tilde{\theta}}}{\cos (\tilde{\theta}-\theta)} \mid \tilde{\theta} \in \overline{\mathcal{D}}\right\} \quad \text { a.s. }
$$

and

$$
\limsup _{n \rightarrow \infty} \frac{\phi(n A, h(n))}{n l(A)}=\inf \left\{\frac{\nu_{\widetilde{\theta}}}{\cos (\widetilde{\theta}-\theta)} \mid \widetilde{\theta} \in \underline{\mathcal{D}}\right\} \quad \text { a.s. }
$$

Corollary 2.5. We suppose that conditions $(\mathbf{H 1}),(\mathbf{H 2})$ and $(\mathbf{H 4})$ on $h$ are satisfied. We suppose also that $(\mathbf{F 2})$ holds. Then we have

$$
\lim _{n \rightarrow \infty} \frac{\phi(n A, h(n))}{n l(A)}=\inf \left\{\frac{\nu_{\tilde{\theta}}}{\cos (\widetilde{\theta}-\theta)} \mid \widetilde{\theta} \in[\theta-\alpha, \theta+\alpha]\right\} \quad \text { in } L^{1} .
$$


Moreover, if 0 is the middle of $A$, or if (F3) holds, then

$$
\lim _{n \rightarrow \infty} \frac{\phi(n A, h(n))}{n l(A)}=\inf \left\{\frac{\nu_{\tilde{\theta}}}{\cos (\tilde{\theta}-\theta)} \mid \widetilde{\theta} \in[\theta-\alpha, \theta+\alpha]\right\} \quad \text { a.s. }
$$

Concerning the lower large deviations of $\tau(n A, h(n))$, Theorem 3.9 and Lemma 5.1 in Rossignol and Théret [14] state that:

Theorem 2.6. For every non-empty line-segment $A$ in $\mathbb{R}^{2}$, with Euclidean length $l(A)$, for every height function $h: \mathbb{N} \rightarrow \mathbb{R}^{+}$satisfying $(\mathbf{H 1})$, for all $\lambda$ in $\mathbb{R}^{+}$, the limit

$$
\mathcal{I}_{\theta}(\lambda)=\lim _{n \rightarrow \infty} \frac{-1}{n l(A)} \log \mathbb{P}\left[\tau(n A, h(n)) \leq\left(\lambda-\frac{1}{\sqrt{n}}\right) n l(A)\right]
$$

exists in $[0,+\infty]$ and depends only on $\theta \in[0, \pi[$ such that $(\cos \theta, \sin \theta)$ is orthogonal to $A$, and not on $h$ nor $A$ itself. Moreover, if the hypotheses $(\mathbf{F 1})$ and $(\mathbf{F 2})$ are satisfied, the function $\mathcal{I}_{\theta}$ has the following properties: it is convex on $\mathbb{R}^{+}$, infinite on $[0, \delta(|\cos \theta|+|\sin \theta|)[$, where $\delta=\inf \{\lambda \mid \mathbb{P}[t(e) \leq \lambda]>0\}$, finite on $] \delta(|\cos \theta|+$ $|\sin \theta|),+\infty\left[\right.$, equal to 0 on $\left[\nu_{\theta},+\infty\left[\right.\right.$, and if $\nu_{\theta}>\delta(|\cos \theta|+|\sin \theta|)$ it is continuous and strictly decreasing on ]$\left.\delta(|\cos \theta|+|\sin \theta|), \nu_{\theta}\right]$ and positive on $] \delta(|\cos \theta|+|\sin \theta|), \nu_{\theta}[$.

For simplicity of notations, we define $\mathcal{I}_{\theta}=+\infty$ on $\mathbb{R}_{*}^{-}$, and for all $a \geq 0$,

$$
\mathcal{I}_{\theta}\left(a^{+}\right)=\lim _{\varepsilon \rightarrow 0, \varepsilon>0} \mathcal{I}_{\theta}(a+\varepsilon) \quad \text { and } \quad \mathcal{I}_{\theta}\left(a^{-}\right)=\lim _{\varepsilon \rightarrow 0, \varepsilon>0} \mathcal{I}_{\theta}(a-\varepsilon)
$$

We denote by $\mathcal{J}_{\theta}$ the function defined on $\mathbb{R}^{+}$by

$$
\mathcal{J}_{\theta}(\lambda)= \begin{cases}\mathcal{I}_{\theta}\left(\lambda^{+}\right) & \text {if } \lambda \leq \nu_{\theta} \\ +\infty & \text { if } \lambda>\nu_{\theta}\end{cases}
$$

The following large deviation principle has also been proved in Rossignol and Théret [14], Theorem 3.10:

Theorem 2.7. For every non-empty line-segment $A$ in $\mathbb{R}^{2}$, with Euclidean length $l(A)$, for every height function $h: \mathbb{N} \rightarrow \mathbb{R}^{+}$satisfying $(\mathbf{H 1})$, if $(\mathbf{F 1})$ and $(\mathbf{F 5})$ hold, then the sequence

$$
\left(\frac{\tau(n A, h(n))}{n l(A)}, n \in \mathbb{N}\right)
$$

satisfies a large deviation principle of speed $n l(A)$ with the good rate function $\mathcal{J}_{\theta}$, where $\theta \in[0, \pi[$ is such that $(\cos \theta, \sin \theta)$ is orthogonal to $A$.

The same large deviation principle is also proved for $\phi(n A, h(n))$ if $\theta=0$ (see Thm. 3.17 in Rossignol and Théret [14], condition (F5) is replaced by (F4) in comparison with Thm. 2.7) or if $h$ satisfies $\lim _{n \rightarrow \infty} h(n) / n=0$ (see Cor. 3.14 in Rossignol and Théret [14]). Theorems 2.6 and 2.7 are valid in any dimension $d \geq 2$. The difference of hypotheses between theorems concerning the variable $\tau$ and theorems concerning the variable $\phi$ will be discussed in Section 2.6.

\subsection{Main results}

As we have seen in Theorem 2.4, the existence of a limit for $\phi(n A, h(n))$ is linked with the equality between 
different infimum. The same holds for the large deviation principle, so we define two additional hypotheses we will use:

\begin{tabular}{|c|}
\hline Hypotheses on $h$ and $F$ \\
\hline (FH1 $) \quad \inf \left\{\frac{\nu_{\tilde{\theta}}}{\cos (\tilde{\theta}-\theta)} \mid \widetilde{\theta} \in \overline{\mathcal{D}}\right\}=\inf \left\{\frac{\nu_{\tilde{\theta}}}{\cos (\tilde{\theta}-\theta)} \mid \widetilde{\theta} \in \underline{\mathcal{D}}\right\}$ \\
$($ FH2 $) \quad \forall \lambda \geq 0, \inf \left\{\frac{1}{\cos (\widetilde{\theta}-\theta)} \mathcal{I}_{\widetilde{\theta}}\left(\lambda \cos (\widetilde{\theta}-\theta)^{+}\right) \mid \widetilde{\theta} \in \operatorname{ad}(\overline{\mathcal{D}})\right\}$ \\
$=\inf \left\{\frac{1}{\cos (\widetilde{\theta}-\theta)} \mathcal{I}_{\widetilde{\theta}}\left(\lambda \cos (\widetilde{\theta}-\theta)^{+}\right) \mid \widetilde{\theta} \in \operatorname{ad}(\underline{\mathcal{D}})\right\}$ \\
\hline
\end{tabular}

Here and in the rest of the paper we denote the adherence of a set $S$ by $\operatorname{ad}(S)$. Notice that $(\mathbf{H 4})$ implies $($ FH1) and (FH2). We can now state our main results:

Theorem 2.8 (lower large deviations). Let $A$ be a non-empty line-segment in $\mathbb{R}^{2}, \theta \in[0, \pi[$ such that $(\cos \theta, \sin \theta)$ is orthogonal to $A$, and $h: \mathbb{N} \rightarrow \mathbb{R}^{+}$satisfying conditions $(\mathbf{H 1})$ and $(\mathbf{H 2})$. If $(\mathbf{F} 1)$, (F2) and (FH1) hold, then for all $\varepsilon>0$ there exist constants $K_{1}(F, A, h, \varepsilon) \geq 0$ and $K_{2}(F, \theta, h, \varepsilon)>0$ such that

$$
\mathbb{P}\left(\frac{\phi(n A, h(n))}{n l(A)} \leq \eta_{\theta, h}-\varepsilon\right) \leq K_{1} \mathrm{e}^{-K_{2} n l(A)},
$$

where

$$
\eta_{\theta, h}=\inf _{\tilde{\theta} \in \overline{\mathcal{D}}} \frac{\nu_{\tilde{\theta}}}{\cos (\widetilde{\theta}-\theta)}=\lim _{n \rightarrow \infty} \frac{\phi(n A, h(n))}{n l(A)} \quad \text { in } L^{1} .
$$

Theorem 2.9 (large deviation principle). Let $A$ be a non-empty line-segment in $\mathbb{R}^{2}, \theta \in[0, \pi[$ such that $(\cos \theta, \sin \theta)$ is orthogonal to $A$, and $h: \mathbb{N} \rightarrow \mathbb{R}^{+}$satisfying conditions $(\mathbf{H 1})$ and $(\mathbf{H 2})$. If (F1), (F2), (FH1), (FH2), and either $(\mathbf{F 4})$ or $(\mathbf{H 3})$ hold, then the sequence

$$
\left(\frac{\phi(n A, h(n))}{n l(A)}, n \in \mathbb{N}\right)
$$

satisfies a large deviation principle of speed $n l(A)$ with the good rate function $\mathcal{K}_{\theta, h}: \mathbb{R}^{+} \rightarrow \mathbb{R}^{+} \cup\{+\infty\}$ defined by

$$
\mathcal{K}_{\theta, h}(\lambda)= \begin{cases}\inf \left\{\frac{1}{\cos (\tilde{\theta}-\theta)} \mathcal{I}_{\tilde{\theta}}\left(\lambda \cos (\tilde{\theta}-\theta)^{+}\right) \mid \tilde{\theta} \in \operatorname{ad}(\overline{\mathcal{D}})\right\} & \text { if } \lambda \leq \eta_{\theta, h} \\ +\infty & \text { if } \lambda>\eta_{\theta, h}\end{cases}
$$

Moreover, if we define

$$
\delta_{\theta, h}=\delta \inf _{\tilde{\theta} \in \overline{\mathcal{D}}} \frac{|\cos \widetilde{\theta}|+|\sin \tilde{\theta}|}{\cos (\widetilde{\theta}-\theta)} .
$$

where $\delta=\inf \{\lambda \mid \mathbb{P}(t(e) \leq \lambda)>0\}$, the good rate function $\mathcal{K}_{\theta, h}$ has the following properties: it is continuous on $\left[0, \eta_{\theta, h}\right]$ except possibly at $\delta_{\theta, h}$ where it may be only right continuous, it is infinite on $\left[0, \delta_{\theta, h}[\cup] \eta_{\theta, h},+\infty[\right.$, finite on $\left.] \delta_{\theta, h}, \eta_{\theta, h}\right]$, positive on $\left[\delta_{\theta, h}, \eta_{\theta, h}\left[\right.\right.$ and equal to 0 at $\eta_{\theta, h}$, and strictly decreasing when it is finite, in the sense that if $\mathcal{K}_{\theta, h}(\lambda)<\infty$, for all $\varepsilon>0, \mathcal{K}_{\theta, h}(\lambda-\varepsilon)>\mathcal{K}_{\theta, h}(\lambda)$.

Remark 2.10. We will prove in Lemma 4.5, Section 4.1 that when $\theta \in\{0, \pi / 2\}$, we have $\mathcal{K}_{\theta, h}(\lambda)=\mathcal{I}_{\theta}\left(\lambda^{+}\right)$, and so Theorem 2.9 is consistent with the large deviation principle obtained in Theorem 3.17 by Rossignol and Théret [14] in the case of straight cylinders.

\subsection{Comments on the hypotheses}

We want to discuss a little bit the different conditions on $F$ and $h$ we use. The condition (H1) is needed to obtain asymptotic results independent of the height function $h$. The condition (F2) is needed to define $\nu_{\theta}$ in the way we did it (it may be relaxed, see Rem. 2.6 in Rossignol and Théret [14]). The condition (F1) is equivalent to the fact that $\nu_{\theta} \neq 0$. 
The conditions (F4) or (H3) appear in Theorem 2.9 to deal with the upper bound of the large deviation principle (see Sect. 4.3.1, in particular Rem. 4.8). They correspond to the condition (F5) in Theorem 2.7. Indeed, we need a stronger moment condition to deal with the upper large deviations of $\tau$ because a minimal cutset corresponding to the maximal flow $\tau(n A, h(n))$ is pinned along the boundary $\partial(n A)$ of $n A$, thus it suffices that some edges in a neighbourhood of this boundary have a huge capacity to increase the variable $\tau(n A, h(n))$ (for more details, see Sect. 2.3 in Théret [18]). Since a minimal cutset corresponding to the flow $\phi(n A, h(n))$ is not pinned, there are no edges in the cylinders with such an influence on $\phi(n A, h(n))$. However, the fact that a cutset for $\phi(n A, h(n))$ is not pinned implies that it can be located anywhere in the cylinder $\operatorname{cyl}(n A, h(n))$, thus we need to control the height of the cylinder by the condition (H2) to obtain interesting results concerning $\phi(n A, h(n))$, whereas this condition does not appear in theorems concerning $\tau(n A, h(n))$. As explained in Remark 3.22 in Rossignol and Théret [14], the condition (H2), combined with (F1), is relevant to observe a maximal flow $\phi(n A, h(n))$ that is not null.

Finally, the conditions (FH1) and (FH2) also appear because the minimal cutset does not have fixed boundary conditions, thus it chooses its orientation to solve an optimisation problem. The condition (FH1) ensures that the direction chosen by an optimal cutset is stable when $n$ goes to infinity; this condition, combined with (F2) and (H1), is relevant to observe a limit for $\phi(n A, h(n)) /(n l(A))$, as proved in Theorem 2.4 . The condition (FH2) is of the same kind, see Section 3.5 for more details. It is not obvious to check if the conditions (FH1) and (FH2) are satisfied, however notice that (H4) implies both (FH1) and (FH2).

\subsection{Outline of the proofs}

For simplicity of notation, we denote by $\phi_{n}$ the maximal flow $\phi(n A, h(n))$ when $A$ and $h$ are clearly given from the context. Here is a detailed table of content of the rest of the article.

Section 3: We prove Theorem 2.8, i.e., that the lower large deviations of $\phi_{n}$ are of surface order.

- 3.1: We prove two technical lemmas on the functions $\left(\mathcal{I}_{\theta}\right)_{\theta \in[0, \pi[}$ : a triangular inequality and a regularity property.

- 3.2: By a subadditive argument (see Fig. 3), we obtain the following large deviation lower bounds (cf. inequalities (3.10) and (3.11)):

$$
\liminf _{n \rightarrow \infty} \frac{1}{n l(A)} \log \mathbb{P}\left[\phi_{n} \leq \lambda n l(A)\right] \geq-\inf _{\widetilde{\theta} \in \operatorname{ad}(\underline{\mathcal{D}})} \frac{1}{\cos (\widetilde{\theta}-\theta)} \mathcal{I}_{\widetilde{\theta}}\left(\lambda \cos (\widetilde{\theta}-\theta)^{-}\right),
$$

and

$$
\limsup _{n \rightarrow \infty} \frac{1}{n l(A)} \log \mathbb{P}\left[\phi_{n} \leq \lambda n l(A)\right] \geq-\inf _{\tilde{\theta} \in \operatorname{ad}(\overline{\mathcal{D}})} \frac{1}{\cos (\widetilde{\theta}-\theta)} \mathcal{I}_{\widetilde{\theta}}\left(\lambda \cos (\widetilde{\theta}-\theta)^{-}\right) .
$$

- 3.3: By a similar subadditive argument (see Fig. 4), we obtain the following large deviation upper bounds (cf. inequalities (3.18) and (3.19)) :

$$
\limsup _{n \rightarrow \infty} \frac{1}{n l(A)} \log \mathbb{P}\left[\phi_{n} \leq \lambda n l(A)\right] \leq-\inf _{\widetilde{\theta} \in \operatorname{ad}(\overline{\mathcal{D}})} \frac{1}{\cos (\widetilde{\theta}-\theta)} \mathcal{I}_{\widetilde{\theta}}\left(\lambda \cos (\widetilde{\theta}-\theta)^{+}\right),
$$

and

$$
\liminf _{n \rightarrow \infty} \frac{1}{n l(A)} \log \mathbb{P}\left[\phi_{n} \leq \lambda n l(A)\right] \leq-\inf _{\widetilde{\theta} \in \operatorname{ad}(\underline{D})} \frac{1}{\cos (\widetilde{\theta}-\theta)} \mathcal{I}_{\widetilde{\theta}}\left(\lambda \cos (\widetilde{\theta}-\theta)^{+}\right) .
$$

- 3.4: From properties of the functions $\left(\mathcal{I}_{\theta}\right)_{\theta \in[0, \pi[}$, we conclude that for all $\lambda<\eta_{\theta, h}$,

$$
\limsup _{n \rightarrow \infty} \frac{1}{n l(A)} \log \mathbb{P}\left[\phi_{n} \leq \lambda n l(A)\right]<0
$$


- 3.5: We discuss the existence of

$$
\lim _{n \rightarrow \infty} \frac{1}{n l(A)} \log \mathbb{P}\left[\phi_{n} \leq \lambda n l(A)\right]
$$

which is linked to condition (FH2).

Section 4: We prove Theorem 2.9, i.e., the large deviation principle itself.

- 4.1: We define the rate function $\mathcal{K}_{\theta, h}$ and study its properties: lower semi-continuity, coercivity, continuity. A deeper understanding of the behaviour of the function $\mathcal{I}_{\theta}$ is needed here. We notice also that for particular orientations $(\theta=k \pi / 4$ for $k \in \mathbb{N})$ we have $\mathcal{K}_{\theta, h}=\mathcal{J}_{\theta}$.

- 4.2: Using classical technics, we prove the lower bound of the large deviation principle.

- 4.3: We prove by imitating technics used in Théret $[15,18]$ that the upper large deviations of $\phi_{n}$ are negligible compared to its lower large deviations. This allows to obtain the upper bound of the large deviation principle, and concludes the proof of Theorem 2.9.

The most original part of our work is the study of the rate function done in Section 4.1. The technics used in Section 3 are largely inspired by the ones in Rossignol and Théret [13], and the proof of the large deviation principle itself in Sections 4.2 and 4.3 is based on quite classical methods.

Finally, we shall use two abbreviations: làglàd for "limite à gauche, limite à droite", meaning that a function admits, on every point of its domain, a limit (eventually infinite) from the left and a limit from the right. We shall also use l.s.c for "lower semi-continuous".

\section{LOWER LARGE DEVIATIONS}

This section is devoted to the study of $\mathbb{P}\left[\phi_{n} \leq \lambda n l(A)\right]$ for $\lambda \geq 0$. We will add conditions on $h$ and $F$ step by step, to emphasize what condition is needed at each time.

\subsection{Technical lemma}

We state here a property which comes from the weak triangle inequality for $\nu$ (see Sect. 4.4 in Rossignol and Théret [14]):

Lemma 3.1. Let $(a b c)$ be a non degenerate triangle in $\mathbb{R}^{2}$ and let $v_{a}, v_{b}, v_{c}$ be the exterior normal unit vectors to the sides $[b c],[a c],[a b]$. We denote by $\left(\cos \theta_{i}, \sin \theta_{i}\right)$ the coordinates of $v_{i}$, and by $l(i j)$ the length of the side $[i, j]$ for $i, j$ in $\{a, b, c\}$. If the angles $\widehat{c a b}$ and $\widehat{a b c}$ have values strictly smaller than $\pi / 2$, then for all $\lambda \geq 0$, for all $\alpha \in[0,1]$, we have

$$
l(a b) \mathcal{I}_{\theta_{c}}\left(\frac{\lambda}{l(a b)}^{+}\right) \leq l(a c) \mathcal{I}_{\theta_{b}}\left(\alpha \frac{\lambda}{l(a c)}^{+}\right)+l(b c) \mathcal{I}_{\theta_{a}}\left((1-\alpha) \frac{\lambda}{l(b c)}^{+}\right)
$$

Proof. This proof follows the one of Proposition 11.6 in Cerf [3]. We consider the cylinder

$$
\operatorname{cyl}_{c}(N)=\operatorname{cyl}(N[a b], N)
$$

of dimensions $N l(a b) \times 2 N$ oriented towards the direction $\theta_{c}$, and we define $\tau_{c}(N)=\tau\left(\operatorname{cyl}_{c}(N)\right)$ (implicitly, for the direction defined by $\theta_{c}$ ). Exactly as in Section 4.1 of Rossignol and Théret [13], we choose two functions $\zeta, h^{\prime}: \mathbb{N} \rightarrow \mathbb{R}^{+}$such that

and

$$
\lim _{n \rightarrow \infty} h^{\prime}(n)=\lim _{n \rightarrow \infty} \zeta(n)=+\infty
$$

$$
\lim _{n \rightarrow \infty} \frac{h^{\prime}(n)}{\zeta(n)}=0
$$




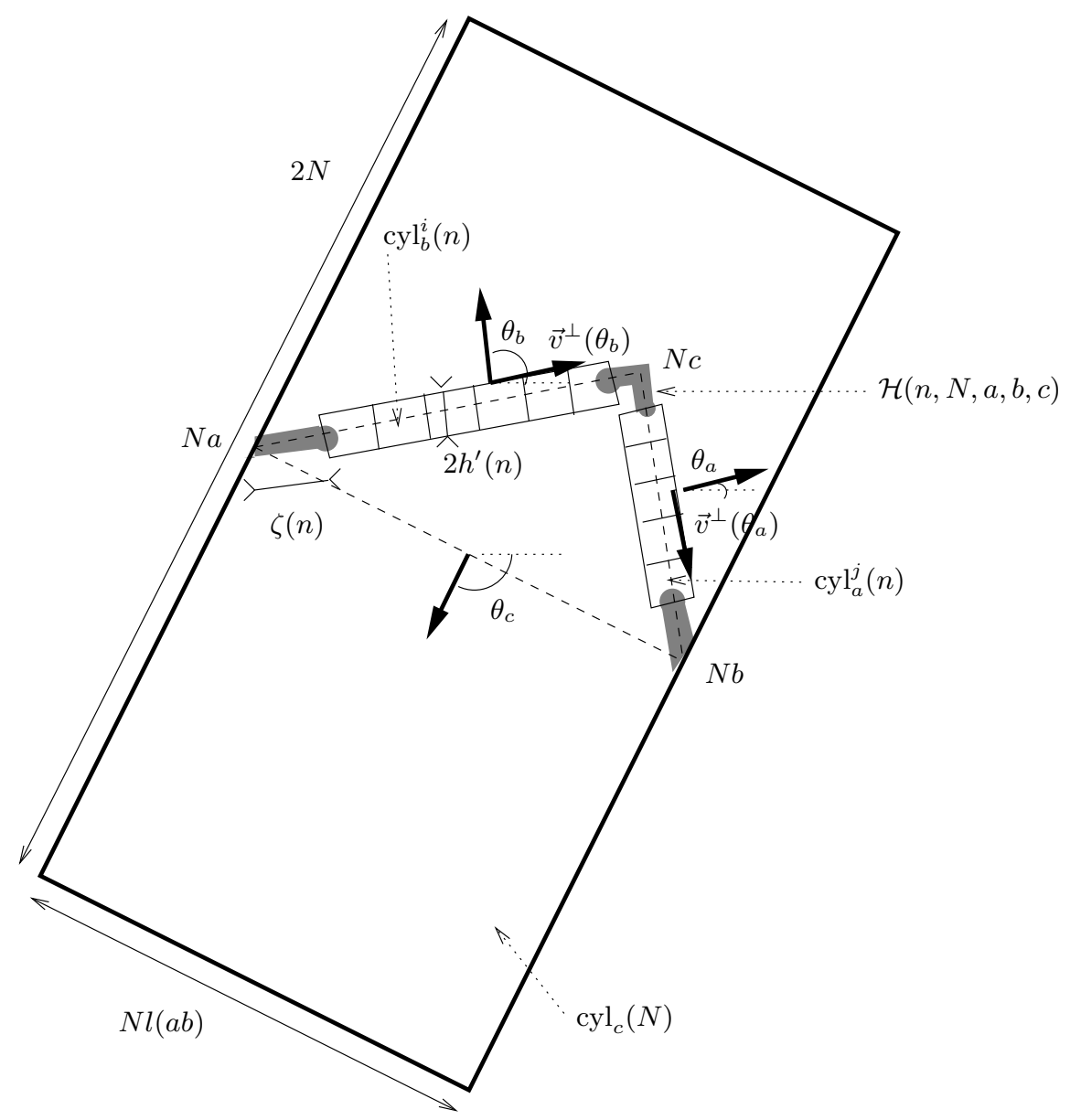

Figure 2. The cylinders $\operatorname{cyl}_{c}(N), \operatorname{cyl}_{b}^{i}(n)$ and $\operatorname{cyl}_{a}^{j}(n)$.

We construct smaller cylinders oriented towards the directions $\theta_{b}$ and $\theta_{a}$ inside $\operatorname{cyl}_{c}(N)$ (see Fig. 2). We define

$$
\begin{aligned}
& \operatorname{cyl}_{b}(n)=\operatorname{cyl}\left(\left[0,0+n l(a b) \vec{v}^{\perp}\left(\theta_{b}\right)\right], h^{\prime}(n)\right), \\
& \operatorname{cyl}_{a}(n)=\operatorname{cyl}\left(\left[0,0+n l(b c) \vec{v}^{\perp}\left(\theta_{a}\right)\right], h^{\prime}(n)\right),
\end{aligned}
$$

respectively oriented towards the direction $\theta_{b}$ and $\theta_{a}$. We define the vectors

$$
\vec{u}_{i}=(\zeta(n)+(i-1) n l(a c)) \vec{v}^{\perp}\left(\theta_{b}\right) \quad \text { and } \quad \vec{w}_{j}=(\zeta(n)+(j-1) n l(b c)) \vec{v}^{\perp}\left(\theta_{a}\right)
$$

and the points

$$
U_{i}=N a+\vec{u}_{i} \quad \text { and } \quad W_{j}=N c+\vec{w}_{j}
$$

for

$$
i \in\left\{1, \ldots, \mathcal{M}_{b}=\left\lfloor\frac{N l(a c)-2 \zeta(n)}{n l(a c)}\right\rfloor\right\} \quad \text { and } \quad j \in\left\{1, \ldots, \mathcal{M}_{a}=\left\lfloor\frac{N l(b c)-2 \zeta(n)}{n l(b c)}\right\rfloor\right\},
$$

where $\mathcal{M}_{a}=\mathcal{M}_{a}(n, N, b, c)$ and $\mathcal{M}_{b}=\mathcal{M}_{b}(n, N, a, c)$. For $i=1, \ldots, \mathcal{M}_{b}\left(\operatorname{resp} . j=1, \ldots, \mathcal{M}_{a}\right)$, let $\widetilde{c y l}_{b}^{i}(n)$ (resp. $\left.\widetilde{\operatorname{cyl}}_{a}^{j}(n)\right)$ be the image of $\operatorname{cyl}_{b}(n)$ (resp. $\left.\operatorname{cyl}_{a}(n)\right)$ the the translation of vector $\overrightarrow{0 U}_{i}\left(\operatorname{resp} \cdot \overrightarrow{0 W}_{j}\right.$ ). We can 
translate again each $\widetilde{c y l}_{b}^{i}(n)$ (resp. $\left.\widetilde{c y l}_{a}^{j}(n)\right)$ by a vector of norm strictly smaller than one to obtain an integer translate $\operatorname{cyl}_{b}^{i}(n)\left(\operatorname{resp} \cdot \operatorname{cyl}_{a}^{j}(n)\right)$ of $\operatorname{cyl}_{b}(n)\left(\right.$ resp. $\left.\operatorname{cyl}_{a}(n)\right)$, i.e., a translate by a vector whose coordinates are in $\mathbb{Z}^{2}$. For $i=1, \ldots, \mathcal{M}_{b}\left(\operatorname{resp} . j=1, \ldots, \mathcal{M}_{a}\right)$, we define $\tau_{b}^{i}(n)=\tau\left(\operatorname{cyl}_{b}^{i}(n)\right)\left(\operatorname{resp} . \tau_{a}^{j}(n)=\tau\left(\operatorname{cyl}_{a}^{j}(n)\right)\right)$ for the direction defined by $\theta_{b}\left(\operatorname{resp} . \theta_{a}\right)$. The dimensions of $\operatorname{cyl}_{b}(n)\left(\operatorname{resp} . \operatorname{cyl}_{a}(n)\right)$ are $(n l(a c)) \times 2 h^{\prime}(n)(\operatorname{resp}$. $\left.(n l(b c)) \times 2 h^{\prime}(n)\right)$, and for $N$ and $n$ large enough cyl $b_{b}^{i}(n)$ and $\operatorname{cyl}_{a}^{j}(n)$ are included in cyl $(N)$ for all $i$ and $j$ (we only consider such large $n$ and $N)$, because $\widehat{c a b}$ and $\widehat{a b c}$ are strictly smaller than $\pi / 2$ and $h^{\prime}(n) / \zeta(n) \rightarrow 0$. The variables $\left(\tau_{b}^{i}(n), \tau_{a}^{j}(n)\right)$ are identically distributed. To glue together cutsets in the cylinders cyl ${ }_{b}^{i}(n)$ and $_{\text {cyl }}^{j}(n)$ for all $i$ and $j$ to obtain a cutset in $\operatorname{cyl}_{c}(N)$ we have to add some edges. We finally define

$$
\mathcal{H}(n, N, a, b, c)=\mathcal{V}\left(\begin{array}{l}
{\left[N a, N a+\vec{u}_{1}\right] \cup\left[N a+\vec{u}_{\mathcal{M}_{b}}, N c\right]} \\
\cup\left[N c, N c+\vec{w}_{1}\right] \cup\left[N c+\vec{w}_{\mathcal{M}_{a}}, N b\right]
\end{array}, 4\right),
$$

and we denote by $H(n, N, a, b, c)$ the set of the edges included in $\mathcal{H}(n, N, a, b, c)$. There exists a constant $C_{1}$ such that

$$
\operatorname{card}(H(n, N, a, b, c)) \leq C_{1}\left(\zeta(n)+n+\frac{N}{n}\right) .
$$

The union of $H(n, N, a, b, c)$ with cutsets in the cylinders $\operatorname{cyl}_{b}^{i}(n)$ and $\operatorname{cyl}_{a}^{j}(n)$ for all $i$ and $j$ separates the upper half part from the lower half part of the boundary of $\operatorname{cyl}_{c}(N)$ (see Fig. 2), so we have

$$
\tau_{c}(N) \leq \sum_{i=1}^{\mathcal{M}_{b}} \tau_{b}^{i}(n)+\sum_{j=1}^{\mathcal{M}_{a}} \tau_{a}^{j}(n)+V(H(n, N, a, b, c))
$$

Then for all $\lambda \geq 0$, for all positive $\eta$, for all large $N$, for all $\alpha \in[0,1]$, by the FKG inequality we have

$$
\begin{aligned}
\mathbb{P}\left[\frac{\tau_{c}(N)}{N l(a b)} \leq\right. & \left.\lambda+3 \eta-\frac{1}{\sqrt{N}}\right] \geq \mathbb{P}\left[\frac{\tau_{c}(N)}{N l(a b)} \leq \lambda+2 \eta\right] \\
\geq & \mathbb{P}\left[\sum_{i=1}^{\mathcal{M}_{b}} \tau_{b}^{i}(n) \leq \alpha(\lambda+\eta) N l(a b)\right] \times \mathbb{P}\left[\sum_{j=1}^{\mathcal{M}_{a}} \tau_{a}^{j}(n) \leq(1-\alpha)(\lambda+\eta) N l(a b)\right] \\
& \times \mathbb{P}[V(H(n, N, a, b, c)) \leq \eta N l(a b)] \\
\geq & \prod_{i=1} \mathbb{P}\left[\tau_{b}^{i}(n) \leq \alpha(\lambda+\eta) n l(a b)\right] \times \prod_{j=1}^{\mathcal{M}_{a}} \mathbb{P}\left[\tau_{a}^{j}(n) \leq(1-\alpha)(\lambda+\eta) n l(a b)\right] \\
& \times \mathbb{P}[V(H(n, N, a, b, c)) \leq \eta N l(a b)] \\
\geq & \mathbb{P}\left[\tau_{a}(n) \leq(1-\alpha)(\lambda+\eta) n l(a b)-\frac{n l(b c)}{\sqrt{n}}\right]^{\mathcal{M}_{a}} \\
\times \mathbb{P}\left[\tau_{b}(n) \leq\right. & \left.\alpha(\lambda+\eta) n l(a b)-\frac{n l(a c)}{\sqrt{n}}\right]^{\mathcal{M}_{b}} \times \mathbb{P}\left[t(e) \leq \frac{\eta N l(a b)}{C_{1}(\zeta(n)+n+N / n)}\right]^{C_{1}(\zeta(n)+n+N / n)}
\end{aligned}
$$

We take the logarithm of the previous inequality, divide it by $-N$, send $N$ to infinity and then $n$ to infinity. We obtain that

$$
l(a b) \mathcal{I}_{\theta_{c}}(\lambda+3 \eta) \leq l(a c) \mathcal{I}_{\theta_{b}}\left(\alpha(\lambda+\eta) \frac{l(a b)}{l(a c)}\right)+l(b c) \mathcal{I}_{\theta_{a}}\left((1-\alpha)(\lambda+\eta) \frac{l(a b)}{l(b c)}\right)
$$

Sending $\eta$ to zero, and replacing $\lambda$ by $\lambda / l(a b)$ we obtain the desired inequality. 
We state next a property of continuity:

Lemma 3.2. For all $\lambda \geq 0$, we define $g_{\lambda}:[\theta-\pi / 2, \theta+\pi / 2] \rightarrow \mathbb{R}^{+} \cup\{+\infty\}$ by

$$
\forall \widetilde{\theta} \in] \theta-\pi / 2, \theta+\pi / 2\left[, \quad g_{\lambda}(\widetilde{\theta})=\frac{1}{\cos (\widetilde{\theta}-\theta)} \mathcal{I}_{\tilde{\theta}}\left(\lambda \cos (\widetilde{\theta}-\theta)^{+}\right)\right.
$$

and

$$
g_{\lambda}(\theta-\pi / 2)=g_{\lambda}(\theta+\pi / 2)= \begin{cases}+\infty & \text { if } \mathcal{I}_{\widetilde{\theta}}\left(0^{+}\right)>0 \\ 0 & \text { if } \mathcal{I}_{\widetilde{\theta}}\left(0^{+}\right)=0\end{cases}
$$

Then $g_{\lambda}$ is lower semi-continuous, and $g_{\lambda}$ is continuous on

$$
H_{\lambda}^{>}=\left\{\tilde{\theta} \mid \lambda>\delta \frac{|\cos \widetilde{\theta}|+|\sin \tilde{\theta}|}{\cos (\widetilde{\theta}-\theta)}\right\} .
$$

Remark 3.3. If $(\mathbf{F 1})$ holds, then for all $\tilde{\theta}$ we have $\nu_{\tilde{\theta}}>0$, that implies $\mathcal{I}_{\tilde{\theta}}\left(0^{+}\right)>0$. The definition of $g_{\lambda}(\theta-\pi / 2)$ and $g_{\lambda}(\theta+\pi / 2)$ is consistent with the expression given for any different $\widetilde{\theta}$. We shall always use Lemma 3.2 under assumption (F1).

Proof. The proof is based on the same ideas as the one of Lemma 3.1, so we will use part of it. We consider two angles $\widetilde{\theta}_{1}, \widetilde{\theta}_{2}$ such that $\widetilde{\theta}_{1}-\widetilde{\theta}_{2}=\hat{\varepsilon}$ (positive or negative) and $|\hat{\varepsilon}|=\varepsilon$ is small. Let (abc) be the right triangle such that, using the same notations as in the previous proof, $l(a b)=1, \theta_{c}=\widetilde{\theta}_{1}+\pi, \theta_{b}=\widetilde{\theta}_{2}$ and $\theta_{a}=\widetilde{\theta}_{2}-\pi / 2$, and so $\widehat{b a c}=\varepsilon, \widehat{a c b}=\pi / 2$ and $\widehat{a b c}<\pi / 2$. Obviously we are confronted with a particular case of triangle ( $a b c$ ) studied in Lemma 3.1. We do exactly the same construction as in the previous proof, and we start again from equation (3.6). Here we have constructed $(a b c)$ such that $l(a b)=1, l(a c)=\cos \varepsilon$ and $l(b c)=\sin \varepsilon$, and by invariance of the graph by a rotation of angle $\pi / 2$, we know that the functions $\mathcal{I}_{\widetilde{\theta}_{2}}$ and $\mathcal{I}_{\widetilde{\theta}_{2}-\pi / 2}$ (respectively $\mathcal{I}_{\widetilde{\theta}_{1}}$ and $\mathcal{I}_{\widetilde{\theta}_{1}+\pi}$ ) are equal. We can rewrite equation (3.6) the following way:

$$
\mathcal{I}_{\widetilde{\theta}_{1}}(\lambda+3 \eta) \leq(\cos \varepsilon) \mathcal{I}_{\widetilde{\theta}_{2}}\left(\alpha \frac{\lambda+\eta}{\cos \varepsilon}\right)+(\sin \varepsilon) \mathcal{I}_{\widetilde{\theta}_{2}}\left((1-\alpha) \frac{\lambda+\eta}{\sin \varepsilon}\right) .
$$

We want to make appear the factor $\cos \left(\widetilde{\theta}_{1}-\theta\right)$, so for all $\lambda \geq 0$ and for all small $\eta$ we deduce from (3.7) that for all $\varepsilon$ small enough,

$$
\begin{aligned}
\mathcal{I}_{\widetilde{\theta}_{1}} & \left(\lambda \cos \left(\widetilde{\theta}_{1}-\theta\right)+3 \eta\right) \\
& \leq(\cos \varepsilon) \mathcal{I}_{\widetilde{\theta}_{2}}\left(\alpha \frac{\lambda \cos \left(\widetilde{\theta}_{1}-\theta\right)+\eta}{\cos \varepsilon}\right)+(\sin \varepsilon) \mathcal{I}_{\widetilde{\theta}_{2}}\left((1-\alpha) \frac{\lambda \cos \left(\widetilde{\theta}_{1}-\theta\right)+\eta}{\sin \varepsilon}\right) \\
& \leq(\cos \varepsilon) \mathcal{I}_{\widetilde{\theta}_{2}}\left(\alpha\left(\lambda \cos \left(\widetilde{\theta}_{2}-\theta\right)+\eta / 2\right)\right)+(\sin \varepsilon) \mathcal{I}_{\widetilde{\theta}_{2}}\left((1-\alpha) \frac{\lambda \cos \left(\widetilde{\theta}_{1}-\theta\right)+\eta}{\sin \varepsilon}\right) .
\end{aligned}
$$

If $\lambda>0$ we choose $\alpha \in] \max (2 / 3,1-\eta /(12 \lambda)), 1[$ (remember that $\lambda$ is fixed and we can choose $\eta$ small in comparison with $\lambda)$, then $\alpha\left(\lambda \cos \left(\widetilde{\theta}_{2}-\theta\right)+\eta / 2\right) \geq \lambda \cos \left(\widetilde{\theta}_{2}-\theta\right)+\eta / 4$. This equation is satisfied for all $1>\alpha \geq 1 / 2$ if $\lambda=0$. We stress here the fact that how large must be $\alpha$ depends on $\lambda$ and $\eta$, but not on $\varepsilon$. With a such fixed big $\alpha$, we obtain that

$$
\begin{aligned}
\mathcal{I}_{\widetilde{\theta}_{1}}\left(\lambda \cos \left(\widetilde{\theta}_{1}-\theta\right)+3 \eta\right) \leq & (\cos \varepsilon) \mathcal{I}_{\widetilde{\theta}_{2}}\left(\lambda \cos \left(\widetilde{\theta}_{2}-\theta\right)+\eta / 4\right) \\
& +(\sin \varepsilon) \mathcal{I}_{\widetilde{\theta}_{2}}\left((1-\alpha) \frac{\lambda \cos \left(\widetilde{\theta}_{1}-\theta\right)+\eta}{\sin \varepsilon}\right) .
\end{aligned}
$$


We send $\widetilde{\theta}_{2}$ to $\widetilde{\theta}_{1}$, i.e. $\varepsilon$ to zero by fixing $\widetilde{\theta}_{1}$. Since $(1-\alpha)\left(\lambda \cos \left(\widetilde{\theta}_{1}-\theta\right)+\eta\right)$ is fixed and positive, we know that for small $\varepsilon$ we obtain

and so for all $\widetilde{\theta}$ we have

$$
(1-\alpha) \frac{\lambda \cos \left(\tilde{\theta}_{1}-\theta\right)+\eta}{\sin \varepsilon}>\nu_{\max }=\max _{\theta \in[0, \pi]} \nu_{\theta},
$$

$$
\mathcal{I}_{\widetilde{\theta}}\left((1-\alpha) \frac{\lambda \cos \left(\tilde{\theta}_{1}-\theta\right)+\eta}{\sin \varepsilon}\right)=0 .
$$

We send finally $\eta$ to zero and obtain

$$
\mathcal{I}_{\widetilde{\theta}_{1}}\left(\lambda \cos \left(\widetilde{\theta}_{1}-\theta\right)^{+}\right) \leq \liminf _{\eta \rightarrow 0} \liminf _{\hat{\varepsilon} \rightarrow 0} \mathcal{I}_{\widetilde{\theta}_{1}+\hat{\varepsilon}}\left(\lambda \cos \left(\widetilde{\theta}_{1}+\hat{\varepsilon}-\theta\right)+\eta / 4\right) .
$$

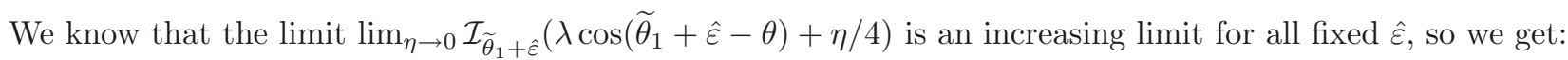

$$
\mathcal{I}_{\widetilde{\theta}_{1}}\left(\lambda \cos \left(\widetilde{\theta}_{1}-\theta\right)^{+}\right) \leq \liminf _{\hat{\varepsilon} \rightarrow 0} \mathcal{I}_{\widetilde{\theta}_{1}+\hat{\varepsilon}}\left(\lambda \cos \left(\widetilde{\theta}_{1}+\hat{\varepsilon}-\theta\right)^{+}\right) .
$$

We will now fix $\widetilde{\theta}_{2}$ and send $\widetilde{\theta}_{1}$ to $\widetilde{\theta}_{2}$. Starting again from (3.6), for all $\beta>0$, for all $\lambda>0$, for all $\left.\widetilde{\theta}_{2} \in\right] \theta-\pi / 2, \theta+\pi / 2\left[\right.$, for all $\eta$ small enough and $\varepsilon$ small (in particular such that $\left.\widetilde{\theta}_{1} \in\right] \theta-\pi / 2, \theta+\pi / 2[$ too), we obtain

$$
\begin{aligned}
\mathcal{I}_{\widetilde{\theta}_{1}} & \left.\lambda \cos \left(\widetilde{\theta}_{1}-\theta\right)+\beta\right) \\
& \leq \mathcal{I}_{\widetilde{\theta}_{1}}\left(\lambda \cos \left(\widetilde{\theta}_{1}-\theta\right)\right) \\
& \leq(\cos \varepsilon) \mathcal{I}_{\widetilde{\theta}_{2}}\left(\alpha \frac{\lambda \cos \left(\widetilde{\theta}_{1}-\theta\right)-2 \eta}{\cos \varepsilon}\right)+(\sin \varepsilon) \mathcal{I}_{\widetilde{\theta}_{2}}\left((1-\alpha) \frac{\lambda \cos \left(\widetilde{\theta}_{1}-\theta\right)-2 \eta}{\sin \varepsilon}\right) \\
& \leq(\cos \varepsilon) \mathcal{I}_{\widetilde{\theta}_{2}}\left(\alpha\left(\lambda \cos \left(\widetilde{\theta}_{2}-\theta\right)-3 \eta\right)\right)+(\sin \varepsilon) \mathcal{I}_{\widetilde{\theta}_{2}}\left((1-\alpha) \frac{\lambda \cos \left(\widetilde{\theta}_{1}-\theta\right)-2 \eta}{\sin \varepsilon}\right) .
\end{aligned}
$$

Exactly as previously, for $\alpha<1$ but sufficiently close to 1 (how close depending on $\lambda$ and $\eta$ but not on $\varepsilon$ ), we have

$$
\begin{aligned}
& \mathcal{I}_{\widetilde{\theta}_{1}}\left(\lambda \cos \left(\widetilde{\theta}_{1}-\theta\right)+\beta\right) \\
& \quad \leq(\cos \varepsilon) \mathcal{I}_{\widetilde{\theta}_{2}}\left(\lambda \cos \left(\widetilde{\theta}_{2}-\varepsilon\right)-4 \eta\right)+(\sin \varepsilon) \mathcal{I}_{\widetilde{\theta}_{2}}\left((1-\alpha) \frac{\lambda \cos \left(\widetilde{\theta}_{1}-\theta\right)-2 \eta}{\sin \varepsilon}\right)
\end{aligned}
$$

We send first $\beta$ to zero, then $\widetilde{\theta}_{1}$ to $\widetilde{\theta}_{2}$ (thus $\varepsilon$ to zero), and finally $\eta$ to zero to obtain as for (3.8) that

$$
\mathcal{I}_{\widetilde{\theta}_{2}}\left(\lambda \cos \left(\widetilde{\theta}_{2}-\theta\right)^{-}\right) \geq \limsup _{\hat{\varepsilon} \rightarrow 0} \mathcal{I}_{\widetilde{\theta}_{2}+\hat{\varepsilon}}\left(\lambda \cos \left(\widetilde{\theta}_{2}+\hat{\varepsilon}-\theta\right)^{+}\right)
$$

This inequality remains valid for $\lambda=0$ or $\cos \left(\widetilde{\theta}_{2}-\theta\right)=0$, since for convenience we decided that $\mathcal{I}_{\widetilde{\theta}_{2}}\left(0^{-}\right)=+\infty$. From (3.8) and (3.9), we conclude that for all $\lambda \geq 0$ :

$$
\begin{aligned}
\frac{1}{\cos (\widetilde{\theta}-\theta)} \mathcal{I}_{\widetilde{\theta}}\left(\lambda \cos (\widetilde{\theta}-\theta)^{+}\right) & \leq \liminf _{\hat{\varepsilon} \rightarrow 0} g_{\lambda}(\widetilde{\theta}+\hat{\varepsilon}) \\
& \leq \limsup _{\hat{\varepsilon} \rightarrow 0} g_{\lambda}(\widetilde{\theta}+\hat{\varepsilon}) \leq \frac{1}{\cos (\widetilde{\theta}-\theta)} \mathcal{I}_{\widetilde{\theta}}\left(\lambda \cos (\widetilde{\theta}-\theta)^{-}\right)
\end{aligned}
$$


Lemma 3.2 follows, since we know that:

$$
\forall \widetilde{\theta} \in H_{\lambda}^{>} \quad \mathcal{I}_{\widetilde{\theta}}\left(\lambda \cos (\widetilde{\theta}-\theta)^{+}\right)=\mathcal{I}_{\widetilde{\theta}}\left(\lambda \cos (\widetilde{\theta}-\theta)^{-}\right) .
$$

\subsection{Lower bound}

From now on, we suppose that the height function $h$ satisfies (H1). We will use equation (19) of Rossignol and Théret [13], and thus the construction that leads to it, to prove that

$$
\liminf _{n \rightarrow \infty} \frac{1}{n l(A)} \log \mathbb{P}\left[\phi_{n} \leq \lambda n l(A)\right] \geq-\inf _{\widetilde{\theta} \in \operatorname{ad}(\underline{\mathcal{D}})} \frac{1}{\cos (\widetilde{\theta}-\theta)} \mathcal{I}_{\widetilde{\theta}}\left(\lambda \cos (\widetilde{\theta}-\theta)^{-}\right),
$$

and

$$
\limsup _{n \rightarrow \infty} \frac{1}{n l(A)} \log \mathbb{P}\left[\phi_{n} \leq \lambda n l(A)\right] \geq-\inf _{\widetilde{\theta} \in \operatorname{ad}(\overline{\mathcal{D}})} \frac{1}{\cos (\widetilde{\theta}-\theta)} \mathcal{I}_{\widetilde{\theta}}\left(\lambda \cos (\widetilde{\theta}-\theta)^{-}\right),
$$

We recall this construction here. We consider a line segment $A$, of orthogonal unit vector $\vec{v}(\theta)=(\cos \theta, \sin \theta)$ for $\theta \in\left[0, \pi\left[\right.\right.$, and a function $h: \mathbb{N} \rightarrow \mathbb{R}^{+}$satisfying $\lim _{n \rightarrow \infty} h(n)=+\infty$. We use the notation $\mathcal{D}_{n}=\mathcal{D}(n A, h(n))$. For all $\widetilde{\theta} \in \mathcal{D}_{n}$, we define

$$
k_{n}=\frac{1}{2}+\frac{n l(A) \tan (\tilde{\theta}-\theta)}{4 h(n)},
$$

and thus $\kappa_{n}=\left(k_{n}, \widetilde{\theta}\right) \in D_{n}$. We want to compare $\phi_{n}^{\kappa_{n}}$ with the maximal flow $\tau$ in a cylinder $\operatorname{inside} \operatorname{cyl}(n A, h(n))$ and oriented towards the direction $\widetilde{\theta}$. In fact, we must use the subadditivity of $\tau$ and compare $\phi_{n}^{\kappa_{n}}$ with a sum of such variables $\tau$.

We consider $n$ and $N$ in $\mathbb{N}$, with $N$ a lot bigger than $n$. The following definitions can seem a little bit complicated, but Figure 3 is more explicit. We choose two functions $h^{\prime}, \zeta: \mathbb{N} \rightarrow \mathbb{R}^{+}$such that

$$
\lim _{n \rightarrow \infty} h^{\prime}(n)=\lim _{n \rightarrow \infty} \zeta(n)=+\infty
$$

and

$$
\lim _{n \rightarrow \infty} \frac{h^{\prime}(n)}{\zeta(n)}=0
$$

We consider a fixed $\tilde{\theta} \in \mathcal{D}_{N}$. We recall that

$$
\vec{v}(\widetilde{\theta})=(\cos \widetilde{\theta}, \sin \widetilde{\theta}) \text { and } \quad \vec{v}^{\perp}(\widetilde{\theta})=(\sin \widetilde{\theta},-\cos \widetilde{\theta}) .
$$

In $\operatorname{cyl}(N A, h(N))$, we denote by $x_{N}$ and $y_{N}$ the two points corresponding to the boundary conditions $\kappa_{N}$, such that $\overrightarrow{x_{N} y_{N}} \cdot \vec{v}^{\perp}(\widetilde{\theta})>0$. Notice that according to our choice of $k_{N}$, the segments $\left[x_{N}, y_{N}\right]$ and $N A$ cut each other in their middle. If we denote by $L(N, \widetilde{\theta})$ the distance between $x_{N}$ and $y_{N}$, we have:

$$
L(N, \widetilde{\theta})=\frac{N l(A)}{\cos (\widetilde{\theta}-\theta)}
$$

We define

$$
\operatorname{cyl}^{\prime}(n)=\operatorname{cyl}\left(\left[0, n \vec{v}^{\perp}(\widetilde{\theta})\right], h^{\prime}(n)\right) .
$$

We will translate $\operatorname{cyl}^{\prime}(n)$ numerous times inside $\operatorname{cyl}(N A, h(N))$. We define

$$
t_{i}=x_{N}+(\zeta(n)+(i-1) n) \vec{v}^{\perp}(\widetilde{\theta})
$$




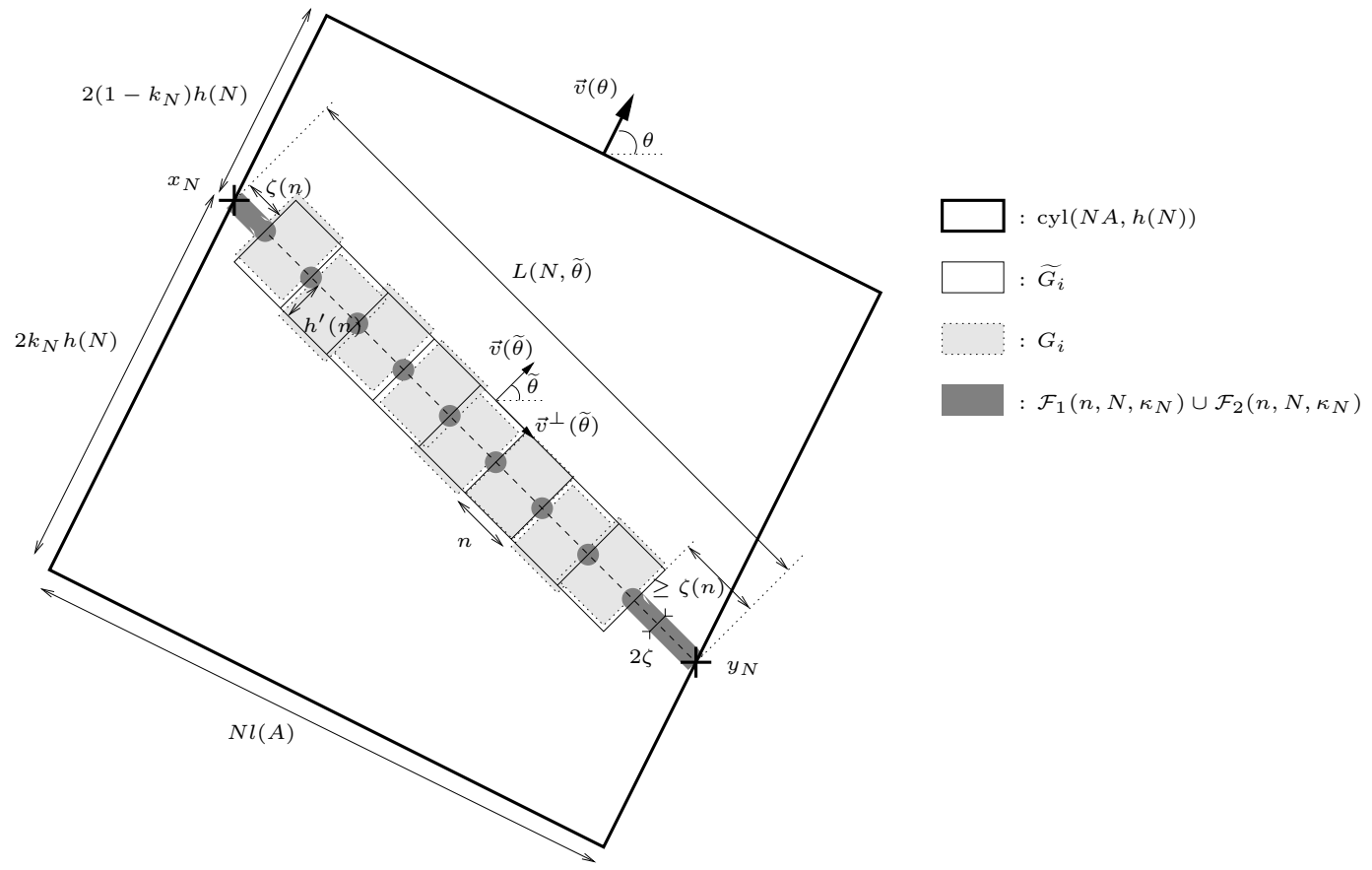

Figure 3 . The cylinders $\operatorname{cyl}(N A, h(N))$ and $G_{i}$, for $i=1, \ldots, \mathcal{M}$.

for $i=1, \ldots, \mathcal{M}$, where

$$
\mathcal{M}=\mathcal{M}(n, N)=\left\lfloor\frac{L(N, \widetilde{\theta})-2 \zeta(n)}{n}\right\rfloor .
$$

Of course we consider only $N$ large enough to have $\mathcal{M} \geq 2$. For $i=1, \ldots, \mathcal{M}$, we denote by $\widetilde{G_{i}}$ the image of $\operatorname{cyl}^{\prime}(n)$ by the translation of vector $\overrightarrow{0 t_{i}}$. For $n$ (and thus $N$ ) sufficiently large, thanks to condition (3.12), we know that $\widetilde{G_{i}} \subset \operatorname{cyl}(N A, h(N))$ for all $i$. We can translate $\widetilde{G_{i}}$ again by a vector of norm strictly smaller than 1 to obtain an integer translate of $\operatorname{cyl}^{\prime}(n)$ (i.e., a translate by a vector whose coordinates are in $\mathbb{Z}^{2}$ ) that we will call $G_{i}$. Now we want to glue together cutsets of boundary condition $(1 / 2, \widetilde{\theta})$ in the cylinders $G_{i}$. We define:

$$
\mathcal{F}_{1}\left(n, N, \kappa_{N}\right)=\left(\bigcup_{i=1}^{\mathcal{M}} \mathcal{V}\left(t_{i}, \zeta_{0}\right)\right) \bigcap \operatorname{cyl}(N A, h(N))
$$

where $\zeta_{0}$ is a fixed constant larger than 4 , and:

$$
\mathcal{F}_{2}\left(n, N, \kappa_{N}\right)=\mathcal{V}\left(\left[x_{N}, x_{N}+\zeta(n) \vec{v}^{\perp}(\widetilde{\theta})\right] \cup\left[z_{\mathcal{M}}, y_{N}\right], \zeta_{0}\right) \bigcap \operatorname{cyl}(N A, h(N)) .
$$

Let $F_{1}\left(n, N, \kappa_{N}\right)$ (respectively $F_{2}\left(n, N, \kappa_{N}\right)$ ) be the set of the edges included in $\mathcal{F}_{1}\left(n, N, \kappa_{N}\right)$ (respectively $\left.\mathcal{F}_{2}\left(n, N, \kappa_{N}\right)\right)$. If for every $i=1, \ldots, \mathcal{M}, \mathcal{G}_{i}$ is a cutset of boundary condition $(1 / 2, \widetilde{\theta})$ in $G_{i}$, then

$$
\bigcup_{i=1}^{\mathcal{M}} \mathcal{G}_{i} \cup F_{1}\left(n, N, \kappa_{N}\right) \cup F_{2}\left(n, N, \kappa_{N}\right)
$$


contains a cutset of boundary conditions $\kappa_{N}$ in $\operatorname{cyl}(N A, h(N))$. We obtain:

$$
\phi_{N}^{\kappa_{N}} \leq \sum_{i=1}^{\mathcal{M}} \tau\left(G_{i}, \vec{v}(\widetilde{\theta})\right)+V\left(F_{1}\left(n, N, \kappa_{N}\right) \cup F_{2}\left(n, N, \kappa_{N}\right)\right),
$$

and so,

$$
\forall \widetilde{\theta} \in \mathcal{D}_{N} \quad \phi_{N} \leq \phi_{N}^{\kappa_{N}} \leq \sum_{i=1}^{\mathcal{M}} \tau\left(G_{i}, \vec{v}(\widetilde{\theta})\right)+V\left(F_{1}\left(n, N, \kappa_{N}\right) \cup F_{2}\left(n, N, \kappa_{N}\right)\right),
$$

This equation (3.14) is equation (19) in Rossignol and Théret [13]. Moreover, there exists a constant $C_{2}$ such that:

$$
\operatorname{card}\left(F_{1}\left(n, N, \kappa_{N}\right)\right) \leq C_{2} \mathcal{M} \text { and } \operatorname{card}\left(F_{2}\left(n, N, \kappa_{N}\right)\right) \leq C_{2}(\zeta(n)+n) .
$$

Then for all $\widetilde{\theta} \in \mathcal{D}_{n}$, for all $\lambda>0$, for all positive small $\varepsilon$, by the FKG inequality,

$$
\begin{aligned}
& \mathbb{P}\left[\phi_{N} \leq \lambda l(A) N\right] \\
& \geq \mathbb{P}\left[\bigcap_{i=1}^{\mathcal{M}}\left\{\tau\left(G_{i}, \vec{v}(\widetilde{\theta})\right) \leq(\lambda-\varepsilon) \frac{N l(A)}{\mathcal{M}}\right\} \cap\left\{V\left(F_{1}\left(n, N, \kappa_{N}\right) \cup F_{2}\left(n, N, \kappa_{N}\right)\right) \leq \varepsilon l(A) N\right\}\right] \\
& \geq \mathbb{P}\left[\tau\left(\operatorname{cyl}^{\prime}(n), \vec{v}(\widetilde{\theta})\right) \leq(\lambda-\varepsilon) \cos (\widetilde{\theta}-\theta) n\right]^{\mathcal{M}} \\
& \times \mathbb{P}\left[\forall e \in F_{1}\left(n, N, \kappa_{N}\right) \cup F_{2}\left(n, N, \kappa_{N}\right), t(e) \leq \frac{\varepsilon l(A) N}{C_{2}(\mathcal{M}+\zeta(n)+n)}\right] \\
& \geq \mathbb{P}\left[\frac{\tau\left(\operatorname{cyl}^{\prime}(n), \vec{v}(\widetilde{\theta})\right)}{n} \leq(\lambda-\varepsilon) \cos (\widetilde{\theta}-\theta)-\frac{1}{\sqrt{n}}\right]^{\mathcal{M}} \\
& \times \mathbb{P}\left[t(e) \leq \frac{\varepsilon l(A) N}{C_{2}(\mathcal{M}+\zeta(n)+n)}\right]^{C_{2}(\mathcal{M}+\zeta(n)+n)} .
\end{aligned}
$$

We take the logarithm of this inequality, divide it by $N l(A)$, send $N$ to infinity and then $n$ to infinity. Thanks to Theorem 2.6, for all $\widetilde{\theta} \in \underline{\mathcal{D}}$ and $\lambda>\varepsilon>0$, we obtain

$$
\liminf _{N \rightarrow \infty} \frac{1}{N l(A)} \log \mathbb{P}\left[\frac{\phi_{N}}{N l(A)} \leq \lambda\right] \geq \frac{-1}{\cos (\widetilde{\theta}-\theta)} \mathcal{I}_{\widetilde{\theta}}((\lambda-\varepsilon) \cos (\widetilde{\theta}-\theta)) .
$$

Sending $\varepsilon$ to zero (remember that $\mathcal{I}_{\widetilde{\theta}}$ is làglàd) and taking the infimum in $\widetilde{\theta}$,

$$
\liminf _{N \rightarrow \infty} \frac{1}{N l(A)} \log \mathbb{P}\left[\phi_{N} \leq \lambda N l(A)\right] \geq-\inf _{\tilde{\theta} \in \underline{\mathcal{D}}} \frac{1}{\cos (\widetilde{\theta}-\theta)} \mathcal{I}_{\widetilde{\theta}}\left(\lambda \cos (\widetilde{\theta}-\theta)^{-}\right) .
$$

Similarly, if $\widetilde{\theta} \in \overline{\mathcal{D}}$, let $\psi: \mathbb{N} \rightarrow \mathbb{N}$ be strictly increasing such that for all $N, \widetilde{\theta} \in \mathcal{D}_{\psi(N)}$. Then we obtain by the same arguments that

$$
\begin{aligned}
\limsup _{N \rightarrow \infty} \frac{1}{N l(A)} \log \mathbb{P}\left[\phi_{N} \leq \lambda N l(A)\right] & \geq \liminf _{N \rightarrow \infty} \frac{1}{\psi(N) l(A)} \log \mathbb{P}\left[\phi_{\psi(N)} \leq \lambda \psi(N) l(A)\right] \\
& \geq-\inf _{\widetilde{\theta} \in \overline{\mathcal{D}}} \frac{1}{\cos (\widetilde{\theta}-\theta)} \mathcal{I}_{\widetilde{\theta}}\left(\lambda \cos (\widetilde{\theta}-\theta)^{-}\right) .
\end{aligned}
$$

These inequalities remain valid for $\lambda=0$, since $\mathcal{I}_{\tilde{\theta}}\left(0^{-}\right)=+\infty$, so equations (3.15) and (3.16) are satisfied for all $\lambda \geq 0$. 
We will transform a little bit inequalities (3.15) and (3.16) to make it more useful for us in the proof of the large deviation principle below. Actually, let us prove that:

$$
\inf _{\widetilde{\theta} \in \mathcal{D}} \frac{1}{\cos (\widetilde{\theta}-\theta)} \mathcal{I}_{\widetilde{\theta}}\left(\lambda \cos (\widetilde{\theta}-\theta)^{-}\right)=\inf _{\widetilde{\theta} \in \operatorname{ad}(\mathcal{D})} \frac{1}{\cos (\widetilde{\theta}-\theta)} \mathcal{I}_{\widetilde{\theta}}\left(\lambda \cos (\widetilde{\theta}-\theta)^{-}\right),
$$

where $\mathcal{D}$ is an interval of $[\theta-\pi / 2, \theta+\pi / 2]$ which is centered at $\theta$ and symmetric with respect to $\theta$ (representing $\overline{\mathcal{D}}$ or $\underline{\mathcal{D}}$ here). As we did previously, we define

$$
H_{\lambda}^{*}=\left\{\widetilde{\theta} \mid \lambda * \delta \frac{|\cos \widetilde{\theta}|+|\sin \widetilde{\theta}|}{\cos (\widetilde{\theta}-\theta)}\right\}
$$

where $*$ represents $<,>, \leq, \geq$ or $=$, and for simplicity of notations we define also:

$$
\widetilde{g}_{\lambda}(\widetilde{\theta})=\frac{1}{\cos (\widetilde{\theta}-\theta)} \mathcal{I}_{\tilde{\theta}}\left(\lambda \cos (\widetilde{\theta}-\theta)^{-}\right) .
$$

The function $\widetilde{g}_{\lambda}$ is infinite on $H_{\lambda}^{\leq}$, and finite, continuous and equal to $g_{\lambda}$ on $H_{\lambda}^{>}$. If $\mathcal{D}$ is included in $H_{\lambda}^{\leq}$, then $\operatorname{ad}(\mathcal{D})$ too because $H_{\lambda}^{\leq}$is closed, and then:

$$
\inf _{\mathcal{D}} \widetilde{g}_{\lambda}=+\infty=\inf _{\operatorname{ad}(\mathcal{D})} \widetilde{g}_{\lambda}
$$

Otherwise, $\mathcal{D} \cap H_{\lambda}^{>}$is non empty, so $\inf _{\mathcal{D}} \widetilde{g}_{\lambda}$ is finite. If $\operatorname{ad}(\mathcal{D}) \neq \mathcal{D}$ (otherwise the result is obvious), then $\mathcal{D}$ is open since it is symmetric with respect to $\theta$, and we denote by $\widetilde{\theta}_{1}$ and $\widetilde{\theta}_{2}$ the two points of $\operatorname{ad}(\mathcal{D}) \backslash \mathcal{D}$. Either $\widetilde{g}_{\lambda}$ is continuous at $\widetilde{\theta}_{1}$ (respectively $\left.\widetilde{\theta}_{2}\right)$, or $\widetilde{g}_{\lambda}\left(\widetilde{\theta}_{1}\right)$ (respectively $\widetilde{g}_{\lambda}\left(\widetilde{\theta}_{2}\right)$ ) is infinite, so

$$
\inf _{\mathcal{D}} \widetilde{g}_{\lambda}=\inf _{\operatorname{ad}(\mathcal{D})} \widetilde{g}_{\lambda}
$$

and equation (3.17) is proved. Inequalities (3.15) and (3.16) are equivalent to (3.10) and (3.11).

\subsection{Upper bound}

We suppose from now on that $h$ satisfies (H1) and (H2). We will use equation (24) in Rossignol and Théret [13], and thus the construction that leads to it, to prove that

$$
\limsup _{n \rightarrow \infty} \frac{1}{n l(A)} \log \mathbb{P}\left[\phi_{n} \leq \lambda n l(A)\right] \leq-\inf _{\widetilde{\theta} \in \operatorname{ad}(\overline{\mathcal{D}})} \frac{1}{\cos (\widetilde{\theta}-\theta)} \mathcal{I}_{\widetilde{\theta}}\left(\lambda \cos (\widetilde{\theta}-\theta)^{+}\right),
$$

and

$$
\liminf _{n \rightarrow \infty} \frac{1}{n l(A)} \log \mathbb{P}\left[\phi_{n} \leq \lambda n l(A)\right] \leq-\inf _{\widetilde{\theta} \in \operatorname{ad}(\underline{\mathcal{D}})} \frac{1}{\cos (\widetilde{\theta}-\theta)} \mathcal{I}_{\widetilde{\theta}}\left(\lambda \cos (\widetilde{\theta}-\theta)^{+}\right),
$$

We recall this construction now. We do the symmetric construction of the one done in Section 3.2. We consider $n$ and $N$ in $\mathbb{N}$ and take $N$ a lot bigger than $n$. We choose functions $\zeta^{\prime}, h^{\prime \prime}: \mathbb{N} \rightarrow \mathbb{R}^{+}$such that

$$
\lim _{n \rightarrow \infty} \zeta^{\prime}(n)=\lim _{n \rightarrow \infty} h^{\prime \prime}(n)=+\infty
$$

and

$$
\lim _{n \rightarrow \infty} \frac{h(n)}{\zeta^{\prime}(n)}=0
$$




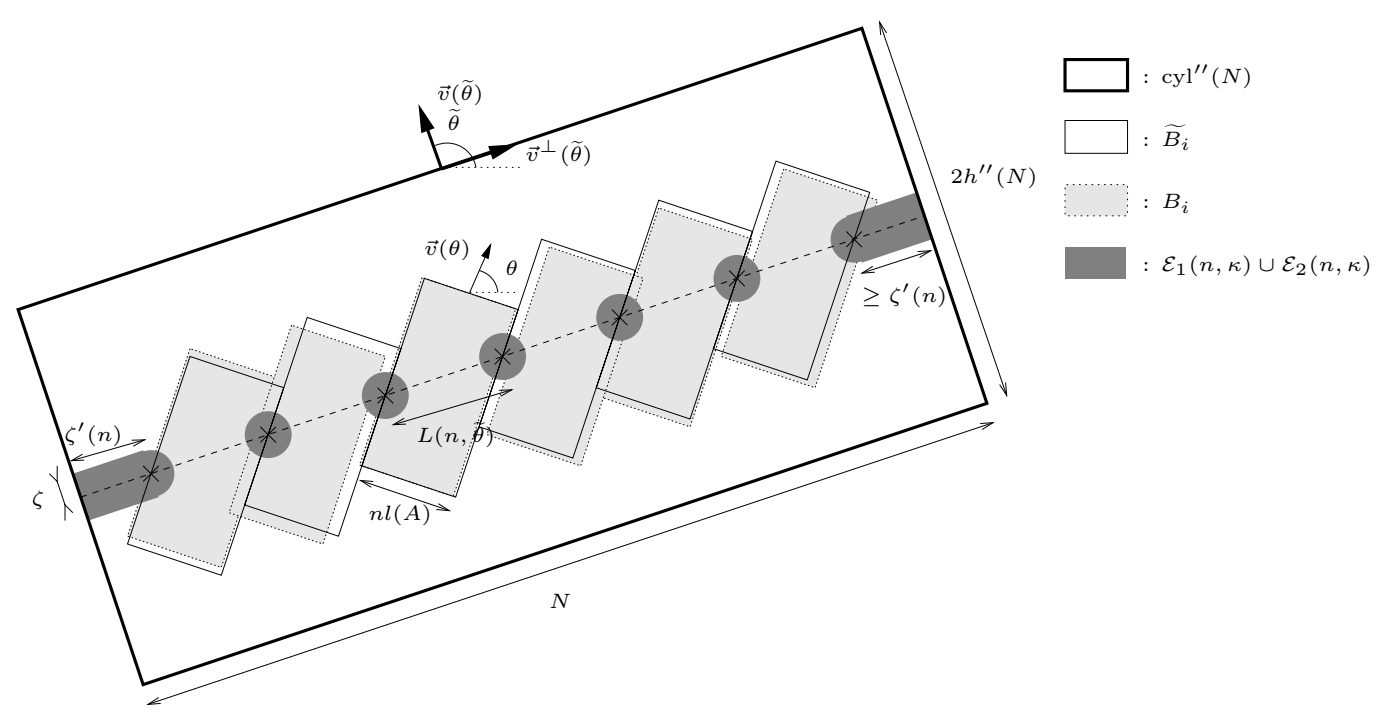

Figure 4 . The cylinders $\operatorname{cyl}^{\prime \prime}(N)$ and $B_{i}$, for $i=1, \ldots, \mathcal{N}$.

We consider $\kappa=(k, \widetilde{\theta}) \in D_{n}$. Keeping the same notations as in Section 3.2, we define

$$
\operatorname{cyl}^{\prime \prime}(N)=\operatorname{cyl}\left(\left[0, N \vec{v}^{\perp}(\widetilde{\theta})\right], h^{\prime \prime}(N)\right) .
$$

We will translate cyl $(n A, h(n))$ numerous times in $\operatorname{cyl}^{\prime \prime}(N)$. Figure 4 is more explicit than the following definitions. The condition $\kappa$ defines two points $x_{n}$ and $y_{n}$ on the boundary of $\operatorname{cyl}(n A, h(n))$ (see Sect. 3.2). As in Section 3.2 , we denote by $L(n, \widetilde{\theta})$ the distance between $x_{n}$ and $y_{n}$, and we have

$$
L(n, \widetilde{\theta})=\frac{n l(A)}{\cos (\widetilde{\theta}-\theta)} .
$$

We define

for $i=1, \ldots, \mathcal{N}$, where

$$
z_{i}=\left(\zeta^{\prime}(n)+(i-1) L(n, \widetilde{\theta})\right) \vec{v}^{\perp}(\widetilde{\theta})
$$

$$
\mathcal{N}=\left\lfloor\frac{N-2 \zeta^{\prime}(n)}{L(n, \widetilde{\theta})}\right\rfloor .
$$

Of course we consider only $N$ large enough to have $\mathcal{N} \geq 2$. For $i=1, \ldots, \mathcal{N}$, we denote by $\widetilde{B_{i}}$ the image of $\operatorname{cyl}(n A, h(n))$ by the translation of vector $\overrightarrow{x_{n} z_{i}}$. For $N$ sufficiently large, thanks to condition (3.20), we know that $\widetilde{B_{i}} \subset \operatorname{cyl}^{\prime \prime}(N)$ for all $i$. We can translate $\widetilde{B}_{i}$ again by a vector of norm strictly smaller than 1 to obtain an integer translate of $\operatorname{cyl}\left(n A, h(n)\right.$ ) (i.e., a translate by a vector whose coordinates are in $\mathbb{Z}^{2}$ ) that we will call $B_{i}$. Now we want to glue together cutsets of boundary condition $\kappa$ in the different $B_{i}$. We define:

$$
\mathcal{E}_{1}(n, N, \kappa)=\left(\bigcup_{i=1}^{\mathcal{N}} \mathcal{V}\left(z_{i}, \zeta\right)\right) \bigcap \operatorname{cyl}^{\prime \prime}(N),
$$

where $\zeta$ is still a fixed constant bigger than 4 , and:

$$
\mathcal{E}_{2}(n, N, \kappa)=\mathcal{V}\left(\left[0, \zeta^{\prime}(n) \vec{v}^{\perp}(\widetilde{\theta})\right] \cup\left[z_{\mathcal{N}}, N \vec{v}^{\perp}(\widetilde{\theta})\right], \zeta\right) \bigcap \operatorname{cyl}^{\prime \prime}(N) .
$$


Let $E_{1}(n, N, \kappa)$ (respectively $\left.E_{2}(n, N, \kappa)\right)$ be the set of the edges included in $\mathcal{E}_{1}(n, N, \kappa)$ (respectively $\left.\mathcal{E}_{2}(n, N, \kappa)\right)$. Then, still by gluing cutsets together, we obtain:

$$
\tau\left(\operatorname{cyl}^{\prime \prime}(N), \vec{v}(\widetilde{\theta})\right) \leq \sum_{i=1}^{\mathcal{N}} \phi^{\kappa}\left(B_{i}, \vec{v}(\theta)\right)+V\left(E_{1}(n, N, \kappa) \cup E_{2}(n, N, \kappa)\right),
$$

This equation (3.21) is equation (24) in Rossignol and Théret [13]. On one hand, there exists a constant $C_{3}$ (independent of $\kappa$ ) such that:

$$
\operatorname{card}\left(E_{1}(n, N, \kappa) \cup E_{2}(n, N, \kappa)\right) \leq C_{3}\left(\mathcal{N}+\zeta^{\prime}(n)+L(n, \widetilde{\theta})\right)
$$

On the other hand, the variables $\left(\phi^{\kappa}\left(B_{i}\right)\right)_{i=1, \ldots, \mathcal{N}}$ are identically distributed, with the same law as $\phi_{n}^{\kappa}$ (because we only consider integer translates). Then for all $\kappa \in D_{n}$, for all $\tilde{\lambda} \geq \varepsilon>0$, for all large $N$, we have by the FKG inequality

$$
\begin{aligned}
& \mathbb{P}\left[\tau\left(\operatorname{cyl}^{\prime \prime}(N), \vec{v}(\widetilde{\theta})\right) \leq\left(\widetilde{\lambda}-\frac{1}{\sqrt{N}}\right) N\right] \geq \mathbb{P}\left[\phi_{n}^{\kappa} \leq(\widetilde{\lambda}-\varepsilon) \frac{N}{\mathcal{N}}\right]^{\mathcal{N}} \times \mathbb{P}\left[V\left(E_{1}(n, \kappa) \cup E_{2}(n, \kappa)\right) \leq \frac{\varepsilon}{2} N\right] \\
& \geq \mathbb{P}\left[\frac{\phi_{n}^{\kappa}}{n l(A)} \leq \frac{\tilde{\lambda}-\varepsilon}{\cos (\widetilde{\theta}-\theta)}\right]^{\mathcal{N}} \times \mathbb{P}\left[t(e) \leq \frac{\varepsilon N}{2 C_{3}\left(\mathcal{N}+\zeta^{\prime}(n)+L(n, \widetilde{\theta})\right)}\right]^{C_{3}\left(\mathcal{N}+\zeta^{\prime}(n)+L(n, \tilde{\theta})\right)} \\
& \quad \geq \mathbb{P}\left[\frac{\phi_{n}^{\kappa}}{n l(A)} \leq \frac{\tilde{\lambda}-\varepsilon}{\cos (\widetilde{\theta}-\theta)}\right]^{\mathcal{N}} \times \mathbb{P}\left[t(e) \leq \frac{\varepsilon l(A) n}{4 C_{3}}\right]^{C_{3}\left(\mathcal{N}+\zeta^{\prime}(n)+L(n, \tilde{\theta})\right)} .
\end{aligned}
$$

We take the logarithm of the previous inequality, divide it by $-N$, and send $N$ to infinity. Thanks to Theorem 2.6 we obtain that:

$$
\mathcal{I}_{\widetilde{\theta}}(\widetilde{\lambda}) \leq \frac{-1}{L(n, \widetilde{\theta})} \log \mathbb{P}\left[\frac{\phi_{n}^{\kappa}}{n l(A)} \leq \frac{\tilde{\lambda}-\varepsilon}{\cos (\widetilde{\theta}-\theta)}\right]-\frac{C_{3}}{L(n, \widetilde{\theta})} \log \mathbb{P}\left[t(e) \leq \frac{\varepsilon l(A) n}{4 C_{3}}\right]
$$

For $n$ large enough,

$$
\mathbb{P}\left[t(e) \leq \frac{\varepsilon l(A) n}{4 C_{3}}\right] \geq \frac{1}{2}
$$

and thus,

$$
\frac{1}{n l(A)} \log \mathbb{P}\left[\frac{\phi_{n}^{\kappa}}{n l(A)} \leq \frac{\widetilde{\lambda}-\varepsilon}{\cos (\widetilde{\theta}-\theta)}\right] \leq-\frac{1}{\cos (\widetilde{\theta}-\theta)} \mathcal{I}_{\widetilde{\theta}}(\widetilde{\lambda})+\frac{C_{4}}{n},
$$

where $C_{4}=C_{4}(l(A))=C_{3} \log 2 / l(A)$. We set $\lambda=(\widetilde{\lambda}-\varepsilon) / \cos (\widetilde{\theta}-\theta)$ (so $\lambda \geq 0$ ), and let $\varepsilon$ go to zero to conclude that for all $\lambda \geq 0$ and $\kappa \in D_{n}$,

$$
\mathbb{P}\left[\frac{\phi_{n}^{\kappa}}{n l(A)} \leq \lambda\right] \leq \exp -\left[n l(A)\left(\frac{1}{\cos (\widetilde{\theta}-\theta)} \mathcal{I}_{\widetilde{\theta}}\left(\lambda \cos (\widetilde{\theta}-\theta)^{+}\right)+\frac{C_{4}}{n}\right)\right] .
$$


We come back now to the study of $\phi_{n}$ itself. From Lemma 2.1 and equation (2.2), we know that there exists a finite subset $\tilde{D}_{n}$ of $D_{n}$ such that $\phi_{n}=\inf _{\kappa \in \tilde{D}_{n}} \phi_{n}^{\kappa}$ and $\operatorname{card}\left(\tilde{D}_{n}\right) \leq C_{0} h(n)^{2}$. Therefore,

$$
\begin{aligned}
\mathbb{P}\left[\phi_{n} \leq \lambda n l(A)\right] & =\mathbb{P}\left[\exists \kappa \in \widetilde{D}_{n} \mid \phi_{n}^{\kappa} \leq \lambda n l(A)\right] \leq \sum_{\kappa \in \widetilde{D}_{n}^{\prime}} \mathbb{P}\left[\phi_{n}^{\kappa} \leq \lambda n l(A)\right] \\
& \leq C_{0} h(n)^{2} \times \max _{\kappa \in D_{n}} \mathbb{P}\left[\phi_{n}^{\kappa} \leq \lambda n l(A)\right] \\
& \leq C_{0} h(n)^{2} \exp \left[-n l(A)\left(\frac{C_{4}}{n}+\inf _{\widetilde{\theta} \in \mathcal{D}_{n}} \frac{1}{\cos (\widetilde{\theta}-\theta)} \mathcal{I}_{\widetilde{\theta}}\left(\lambda \cos (\widetilde{\theta}-\theta)^{+}\right)\right)\right]
\end{aligned}
$$

Since $h$ satisfies (H2), we obtain that:

$$
\limsup _{n \rightarrow \infty} \frac{1}{n l(A)} \log \mathbb{P}\left[\phi_{n} \leq \lambda n l(A)\right] \leq-\liminf _{n \rightarrow \infty} \inf _{\widetilde{\theta} \in \mathcal{D}_{n}} \frac{1}{\cos (\widetilde{\theta}-\theta)} \mathcal{I}_{\widetilde{\theta}}\left(\lambda \cos (\widetilde{\theta}-\theta)^{+}\right),
$$

and

$$
\liminf _{n \rightarrow \infty} \frac{1}{n l(A)} \log \mathbb{P}\left[\phi_{n} \leq \lambda n l(A)\right] \leq-\limsup _{n \rightarrow \infty} \inf _{\widetilde{\theta} \in \mathcal{D}_{n}} \frac{1}{\cos (\widetilde{\theta}-\theta)} \mathcal{I}_{\widetilde{\theta}}\left(\lambda \cos (\widetilde{\theta}-\theta)^{+}\right),
$$

We can now apply Lemmas 4.1 and 4.2 in Rossignol and Théret [13] with $f=g_{\lambda}$, that we know to be l.s.c. thanks to Lemma 3.2, to obtain that

$$
\liminf _{n \rightarrow \infty} \inf _{\tilde{\theta} \in \mathcal{D}_{n}} g_{\lambda}(\widetilde{\theta}) \geq \inf _{\tilde{\theta} \in \operatorname{ad}(\overline{\mathcal{D}})} g_{\lambda}(\widetilde{\theta}) \quad \text { and } \quad \limsup _{n \rightarrow \infty} \inf _{\tilde{\theta} \in \mathcal{D}_{n}} \geq \inf _{\tilde{\theta} \in \operatorname{ad}(\underline{\mathcal{D}})} g_{\lambda}(\widetilde{\theta})
$$

So (3.23) and (3.24) lead to (3.18) and (3.19).

\subsection{Positivity and proof of Theorem $\mathbf{2 . 8}$}

We need some extra hypotheses: from now on, we suppose that (H1), (H2), (F1), (F2) and (FH1) hold. We define

$$
\eta_{\theta, h}=\inf _{\widetilde{\theta} \in \overline{\mathcal{D}}} \frac{\nu_{\tilde{\theta}}}{\cos (\widetilde{\theta}-\theta)}=\inf _{\widetilde{\theta} \in \underline{\mathcal{D}}} \frac{\nu_{\tilde{\theta}}}{\cos (\widetilde{\theta}-\theta)} .
$$

By Theorem 2.4,

$$
\lim _{n \rightarrow \infty} \frac{\phi_{n}}{n l(A)}=\eta_{\theta, h} \quad \text { in } L^{1}
$$

and by Proposition 2.3, $\eta_{\theta, h}>0$. In fact, the convergence of $\phi_{n} /(n l(A))$ in $L^{1}$ is stated in Corollary 2.5 under the stronger assumption (H3). However, the methods used in Rossignol and Théret [14] to prove this $L^{1}$-convergence do not use the condition (H3) itself, and can be performed under the assumption (FH1) instead.

We define

$$
\widetilde{\mathcal{K}}_{\theta, h}(\lambda)=\inf _{\widetilde{\theta} \in \operatorname{ad}(\overline{\mathcal{D}})} \frac{1}{\cos (\widetilde{\theta}-\theta)} \mathcal{I}_{\widetilde{\theta}}\left(\lambda \cos (\widetilde{\theta}-\theta)^{+}\right)=\inf _{\widetilde{\theta} \in \operatorname{ad}(\overline{\mathcal{D}})} g_{\lambda}(\widetilde{\theta}) .
$$

We want to prove that $\widetilde{\mathcal{K}}_{\theta, h}>0$ on $\left[0, \eta_{\theta, h}[\right.$. Indeed, we know that

$$
\limsup _{n \rightarrow \infty} \frac{1}{n l(A)} \log \mathbb{P}\left[\phi_{n} \leq \lambda n l(A)\right] \leq-\widetilde{\mathcal{K}}_{\theta, h}
$$

thus proving that $\widetilde{\mathcal{K}}_{\theta, h}>0$ on $\left[0, \eta_{\theta, h}\right.$ [ is equivalent to proving that the lower large deviations of $\phi_{n} /(n l(A))$ are (at least) of surface order. 
Known properties of $\mathcal{I}_{\widetilde{\theta}}$ are recalled in Theorem 2.6. Let us define

$$
\delta_{\theta, h}=\delta \times \inf _{\tilde{\theta} \in \overline{\mathcal{D}}} \frac{|\cos \widetilde{\theta}|+|\sin \widetilde{\theta}|}{\cos (\widetilde{\theta}-\theta)}
$$

the infimum of the values that $\phi_{n} /(n l(A))$ can take asymptotically. Notice that by continuity of the function whose infimum we consider, $\delta_{\theta, h}$ satisfies

$$
\delta_{\theta, h}=\delta \times \inf _{\widetilde{\theta} \in \operatorname{ad}(\overline{\mathcal{D}})} \frac{|\cos \widetilde{\theta}|+|\sin \widetilde{\theta}|}{\cos (\widetilde{\theta}-\theta)}
$$

Then by Theorem 2.6, $\widetilde{\mathcal{K}}_{\theta, h}$ is infinite on $\left[0, \delta_{\theta, h}[\right.$ and finite on $] \delta_{\theta, h},+\infty\left[\right.$ (its behaviour at $\delta_{\theta, h}$ will be studied in Section 4.1), and $\widetilde{\mathcal{K}}_{\theta, h}$ is null on $\left[\eta_{\theta, h},+\infty\right.$. Since for all $\tilde{\theta}, \lambda \rightarrow \mathcal{I}_{\widetilde{\theta}}\left(\lambda^{+}\right)$is non increasing, so is $\widetilde{\mathcal{K}}_{\theta, h}$ on $\mathbb{R}^{+}$. We state the following result:

Lemma 3.4. The function $\widetilde{\mathcal{K}}_{\theta, h}$ is strictly decreasing on $\left[\delta_{\theta, h}, \eta_{\theta, h}\right]$, i.e.,

$$
\left.\forall \lambda \in] \delta_{\theta, h}, \eta_{\theta, h}\right], \forall \varepsilon>0 \quad \widetilde{\mathcal{K}}_{\theta, h}(\lambda)<\widetilde{\mathcal{K}}_{\theta, h}(\lambda-\varepsilon)
$$

We immediately notice that this lemma implies the positivity of $\widetilde{\mathcal{K}}_{\theta, h}$ on $\left[0, \eta_{\theta, h}[\right.$, and thus Theorem 2.8 through inequality $(3.23)$.

Proof. Thanks to Lemma 3.2, we know that for every fixed $\lambda, \widetilde{\theta} \mapsto g_{\lambda}(\widetilde{\theta})$ is l.s.c. $\operatorname{Since} \operatorname{ad}(\overline{\mathcal{D}})$ is compact, $\inf _{\widetilde{\theta} \in \operatorname{ad}(\overline{\mathcal{D}})} g_{\lambda}(\widetilde{\theta})$ is reached at some $\widetilde{\theta}_{\lambda} \in \operatorname{ad}(\overline{\mathcal{D}})$. Notice also that for every fixed $\widetilde{\theta}, \lambda \mapsto g_{\lambda}(\widetilde{\theta})$ is strictly decreasing (in the same meaning as in Lem. 3.4) on the interval:

$$
\left[\delta \frac{|\cos \widetilde{\theta}|+|\sin \widetilde{\theta}|}{\cos (\widetilde{\theta}-\theta)}, \frac{\nu_{\tilde{\theta}}}{\cos (\widetilde{\theta}-\theta)}\right]
$$

We consider $\left.\lambda \in] \delta_{\theta, h}, \eta_{\theta, h}\right]$. Thus $\widetilde{\mathcal{K}}_{\theta, h}(\lambda)<\infty$, so we can suppose that $\widetilde{\mathcal{K}}_{\theta, h}(\lambda-\varepsilon)<\infty$ otherwise the result is obvious. The condition $\widetilde{\mathcal{K}}_{\theta, h}(\lambda-\varepsilon)<\infty$ is equivalent by definition of $\widetilde{\theta}_{\lambda-\varepsilon}$ to $g_{\lambda-\varepsilon}\left(\widetilde{\theta}_{\lambda-\varepsilon}\right)<\infty$, which implies that

$$
\lambda>\delta \frac{\left|\cos \widetilde{\theta}_{\lambda-\varepsilon}\right|+\left|\sin \widetilde{\theta}_{\lambda-\varepsilon}\right|}{\cos \left(\widetilde{\theta}_{\lambda-\varepsilon}-\theta\right)} .
$$

We deduce that

$$
\left.\lambda \in] \delta \frac{\left|\cos \widetilde{\theta}_{\lambda-\varepsilon}\right|+\left|\sin \widetilde{\theta}_{\lambda-\varepsilon}\right|}{\cos \left(\widetilde{\theta}_{\lambda-\varepsilon}-\theta\right)}, \frac{\nu_{\widetilde{\theta}_{\lambda-\varepsilon}}}{\cos \left(\widetilde{\theta}_{\lambda-\varepsilon}-\theta\right)}\right],
$$

thus

$$
g_{\lambda}\left(\widetilde{\theta}_{\lambda-\varepsilon}\right)<g_{\lambda-\varepsilon}\left(\widetilde{\theta}_{\lambda-\varepsilon}\right) .
$$

We obtain

$$
\widetilde{\mathcal{K}}_{\theta, h}(\lambda)=\inf _{\tilde{\theta} \in \operatorname{ad}(\overline{\mathcal{D}})} g_{\lambda}(\widetilde{\theta}) \leq g_{\lambda}\left(\widetilde{\theta}_{\lambda-\varepsilon}\right)<g_{\lambda-\varepsilon}\left(\widetilde{\theta}_{\lambda-\varepsilon}\right)=\inf _{\tilde{\theta} \in \operatorname{ad}(\overline{\mathcal{D}})} g_{\lambda-\varepsilon}(\widetilde{\theta})=\widetilde{\mathcal{K}}_{\theta, h}(\lambda-\varepsilon) .
$$




\subsection{Discussion}

Combining the results of the two previous sections, we obtain that for all $\lambda \geq 0$, if we define

$$
\square_{n}=\frac{1}{n l(A)} \log \mathbb{P}\left[\phi_{n} \leq \lambda n l(A)\right]
$$

we have

$$
\left\{\begin{array}{l}
-\inf _{\tilde{\theta} \in \operatorname{ad}(\underline{\mathcal{D}})} \frac{\mathcal{I}_{\widetilde{\theta}}\left(\lambda \cos (\widetilde{\theta}-\theta)^{-}\right)}{\cos (\widetilde{\theta}-\theta)} \leq \liminf _{n \rightarrow \infty} \square_{n} \leq-\inf _{\widetilde{\theta} \in \operatorname{ad}(\underline{\mathcal{D}})} \frac{\mathcal{I}_{\widetilde{\theta}}\left(\lambda \cos (\widetilde{\theta}-\theta)^{+}\right)}{\cos (\widetilde{\theta}-\theta)}, \\
-\inf _{\tilde{\theta} \in \operatorname{ad}(\overline{\mathcal{D}})} \frac{\mathcal{I}_{\widetilde{\theta}}\left(\lambda \cos (\widetilde{\theta}-\theta)^{-}\right)}{\cos (\widetilde{\theta}-\theta)} \leq \limsup _{n \rightarrow \infty} \square_{n} \leq-\inf _{\widetilde{\theta} \in \operatorname{ad}(\overline{\mathcal{D}})} \frac{\mathcal{I}_{\widetilde{\theta}}\left(\lambda \cos (\widetilde{\theta}-\theta)^{+}\right)}{\cos (\widetilde{\theta}-\theta)} .
\end{array}\right.
$$

In fact, we will prove in Section 4.1 that for all

$$
\lambda \neq \delta \inf _{\tilde{\theta} \in \overline{\mathcal{D}}} \frac{|\cos \widetilde{\theta}|+|\sin \widetilde{\theta}|}{\cos (\widetilde{\theta}-\theta)}=\delta_{\theta, h}
$$

we have

$$
\inf _{\tilde{\theta} \in \operatorname{ad}(\overline{\mathcal{D}})} \frac{\mathcal{I}_{\widetilde{\theta}}\left(\lambda \cos (\widetilde{\theta}-\theta)^{+}\right)}{\cos (\widetilde{\theta}-\theta)}=\inf _{\tilde{\theta} \in \operatorname{ad}(\overline{\mathcal{D}})} \frac{\mathcal{I}_{\widetilde{\theta}}\left(\lambda \cos (\widetilde{\theta}-\theta)^{-}\right)}{\cos (\widetilde{\theta}-\theta)},
$$

(see the proof of the continuity of $\widetilde{\mathcal{K}}_{\theta, h}$, Lem. 4.2), and similarly for all

$$
\lambda \neq \delta \inf _{\widetilde{\theta} \in \underline{\mathcal{D}}} \frac{|\cos \widetilde{\theta}|+|\sin \widetilde{\theta}|}{\cos (\widetilde{\theta}-\theta)}=\widehat{\delta}_{\theta, h}
$$

we have

It implies that for all $\lambda \neq \delta_{\theta, h}$,

$$
\inf _{\tilde{\theta} \in \operatorname{ad}(\underline{\mathcal{D}})} \frac{\mathcal{I}_{\widetilde{\theta}}\left(\lambda \cos (\tilde{\theta}-\theta)^{+}\right)}{\cos (\widetilde{\theta}-\theta)}=\inf _{\tilde{\theta} \in \operatorname{ad}(\underline{\mathcal{D}})} \frac{\mathcal{I}_{\widetilde{\theta}}\left(\lambda \cos (\tilde{\theta}-\theta)^{-}\right)}{\cos (\widetilde{\theta}-\theta)} .
$$

$$
\liminf _{n \rightarrow \infty} \square_{n}=-\inf _{\tilde{\theta} \in \operatorname{ad}(\underline{\mathcal{D}})} \frac{1}{\cos (\widetilde{\theta}-\theta)} \mathcal{I}_{\widetilde{\theta}}\left(\lambda \cos (\widetilde{\theta}-\theta)^{+}\right)=-\inf _{\tilde{\theta} \in \operatorname{ad}(\underline{\mathcal{D}})} \frac{1}{\cos (\widetilde{\theta}-\theta)} \mathcal{I}_{\widetilde{\theta}}\left(\lambda \cos (\widetilde{\theta}-\theta)^{-}\right)
$$

and for all $\lambda \neq \widehat{\delta}_{\theta, h}$

$$
\limsup _{n \rightarrow \infty} \square_{n}=-\inf _{\widetilde{\theta} \in \operatorname{ad}(\overline{\mathcal{D}})} \frac{1}{\cos (\widetilde{\theta}-\theta)} \mathcal{I}_{\widetilde{\theta}}\left(\lambda \cos (\widetilde{\theta}-\theta)^{+}\right)=-\inf _{\widetilde{\theta} \in \operatorname{ad}(\overline{\mathcal{D}})} \frac{1}{\cos (\widetilde{\theta}-\theta)} \mathcal{I}_{\widetilde{\theta}}\left(\lambda \cos (\widetilde{\theta}-\theta)^{-}\right) .
$$

Under condition (FH2), we have $\delta_{\theta, h}=\widehat{\delta}_{\theta, h}$, since

$$
\begin{aligned}
\delta_{\theta, h} & =\inf \left\{\lambda \in \mathbb{R}^{+} \mid \inf _{\widetilde{\theta} \in \operatorname{ad}(\overline{\mathcal{D}})} \frac{\mathcal{I}_{\widetilde{\theta}}\left(\lambda \cos (\widetilde{\theta}-\theta)^{+}\right)}{\cos (\widetilde{\theta}-\theta)}(\lambda)<\infty\right\} \\
& =\inf \left\{\lambda \in \mathbb{R}^{+} \mid \inf _{\tilde{\theta} \in \operatorname{ad}(\underline{\mathcal{D}})} \frac{\mathcal{I}_{\widetilde{\theta}}\left(\lambda \cos (\widetilde{\theta}-\theta)^{+}\right)}{\cos (\widetilde{\theta}-\theta)}(\lambda)<\infty\right\}=\widehat{\delta}_{\theta, h} .
\end{aligned}
$$


Thus, under condition (FH2), we obtain that for all $\lambda \neq \delta_{\theta, h}$, we know that $\lim _{n \rightarrow \infty} \square_{n}$ exists and

$$
\lim _{n \rightarrow \infty} \frac{1}{n l(A)} \log \mathbb{P}\left[\phi_{n} \leq \lambda n l(A)\right]=\widetilde{\mathcal{K}}_{\theta, h}(\lambda) .
$$

Notice that if $\mathcal{I}_{\widetilde{\theta}_{0}}\left(\delta\left(\left|\cos \widetilde{\theta}_{0}\right|+\left|\sin \widetilde{\theta}_{0}\right|\right)^{+}\right)<\infty$ for some $\widetilde{\theta}_{0} \in \overline{\mathcal{D}}$ such that $\delta_{\theta, h}=\delta\left(\left|\cos \widetilde{\theta}_{0}\right|+\left|\sin \widetilde{\theta}_{0}\right|\right) / \cos \left(\widetilde{\theta}_{0}-\theta\right)$, then

$$
-\infty=-\inf _{\tilde{\theta} \in \overline{\mathcal{D}}} \frac{1}{\cos (\widetilde{\theta}-\theta)} \mathcal{I}_{\widetilde{\theta}}\left(\delta_{\theta, h} \cos (\widetilde{\theta}-\theta)^{-}\right)<-\inf _{\tilde{\theta} \in \overline{\mathcal{D}}} \frac{1}{\cos (\widetilde{\theta}-\theta)} \mathcal{I}_{\widetilde{\theta}}\left(\delta_{\theta, h} \cos (\widetilde{\theta}-\theta)^{+}\right),
$$

thus we have no hope to prove equation (3.25) at the point $\lambda=\delta_{\theta, h}$.

\section{LARGE DEVIATION PRINCIPLE}

From now on, we suppose that hypotheses $(\mathbf{H 1}),(\mathbf{H} 2),(\mathbf{F 1}),(\mathbf{F} 2),(\mathbf{F H 1})$ and $(\mathbf{F H 2})$ hold (we recall that (H4) implies (FH1) and (FH2)). By definition, we have

$$
\begin{aligned}
\forall \lambda \in \mathbb{R}^{+}, \widetilde{\mathcal{K}}_{\theta, h}(\lambda) & =\inf _{\tilde{\theta} \in \operatorname{ad}(\overline{\mathcal{D}})} \frac{1}{\cos (\widetilde{\theta}-\theta)} \mathcal{I}_{\widetilde{\theta}}\left(\lambda \cos (\widetilde{\theta}-\theta)^{+}\right) \\
& =\inf _{\tilde{\theta} \in \operatorname{ad}(\underline{\mathcal{D}})} \frac{1}{\cos (\widetilde{\theta}-\theta)} \mathcal{I}_{\widetilde{\tilde{\theta}}}\left(\lambda \cos (\widetilde{\theta}-\theta)^{+}\right) .
\end{aligned}
$$

We define the rate function $\mathcal{K}_{\theta, h}: \mathbb{R}^{+} \rightarrow \mathbb{R}^{+} \cup\{+\infty\}$ by:

$$
\mathcal{K}_{\theta, h}(\lambda)= \begin{cases}\inf _{\tilde{\theta} \in \operatorname{ad}(\overline{\mathcal{D}})} \frac{1}{\cos (\widetilde{\theta}-\theta)} \mathcal{I}_{\widetilde{\theta}}\left(\lambda \cos (\widetilde{\theta}-\theta)^{+}\right)=\widetilde{\mathcal{K}}_{\theta, h}(\lambda) & \text { if } \lambda \leq \eta_{\theta, h}, \\ +\infty & \text { if } \lambda>\eta_{\theta, h} .\end{cases}
$$

\subsection{Properties of $\mathcal{K}_{\theta, h}$}

We can deduce a lot of properties of $\mathcal{K}_{\theta, h}$ from the properties of $\widetilde{\mathcal{K}}_{\theta, h}$ stated in Section 3.4: $\mathcal{K}_{\theta, h}$ is infinite on $\left[0, \delta_{\theta, h}\right.$ [ (if $\delta_{\theta, h}>0$ ) and on $] \eta_{\theta, h},+\infty$ [, finite on $\left.] \delta_{\theta, h}, \eta_{\theta, h}\right]$ (if $\eta_{\theta, h}>\delta_{\theta, h}$ ) and strictly decreasing on $\left[\delta_{\theta, h}, \eta_{\theta, h}\right]$ in the sense of Lemma 3.4. We only have to prove that $\mathcal{K}_{\theta, h}$ is a good rate function, and that it is continuous on $\left[0, \eta_{\theta, h}\right]$ except possibly at $\delta_{\theta, h}$ where it may be only right continuous. We first state that $\mathcal{K}_{\theta, h}$ is a good rate function:

Lemma 4.1. The function $\mathcal{K}_{\theta, h}$ is lower semi-continuous and coercive on $\mathbb{R}^{+}$, i.e., for all $t \geq 0$, the set $\left\{\lambda \mid \mathcal{K}_{\theta, h}(\lambda) \leq t\right\}$ is compact.

We will use this property to prove that $\widetilde{\mathcal{K}}_{\theta, h}$ is right continuous.

Proof. In fact it is sufficient to prove that for all $t \geq 0$, the set $\left\{\lambda \mid \widetilde{\mathcal{K}}_{\theta, h}(\lambda) \leq t\right\}$ is closed, because we know that

$$
\forall t \geq 0 \quad\left\{\lambda \mid \mathcal{K}_{\theta, h}(\lambda) \leq t\right\}=\left\{\lambda \mid \widetilde{\mathcal{K}}_{\theta, h}(\lambda) \leq t\right\} \cap[0, \eta(\theta, h)] .
$$

Let $\left(\lambda_{n}\right)_{n \geq 0}$ be a sequence of $\left\{\lambda \mid \widetilde{\mathcal{K}}_{\theta, h}(\lambda) \leq t\right\}$, converging towards some $\lambda_{0}$. For each fixed $\lambda$ in $\mathbb{R}^{+}$, since the function $g_{\lambda}$ is lower semi-continuous and $\operatorname{ad}(\overline{\mathcal{D}})$ is compact, there exists $\widetilde{\theta}_{\lambda}$ such that

$$
\widetilde{\mathcal{K}}_{\theta, h}(\lambda)=g_{\lambda}\left(\widetilde{\theta}_{\lambda}\right) .
$$

The sequence $\left(\widetilde{\theta}_{\lambda_{n}}\right)_{n \geq 0}$ takes values in the compact ad $(\overline{\mathcal{D}})$, so up to extracting a subsequence, we can suppose that it converges towards a limit $\widetilde{\theta}_{0} \in \operatorname{ad}(\overline{\mathcal{D}})$. For all positive $\varepsilon$, for all large $n$ we have $\lambda_{n} \leq \lambda_{0}+\varepsilon$, and so, 
since $\mathcal{I}_{\widetilde{\theta}}$ is non increasing for all $\widetilde{\theta}$, we obtain for all large $n$ that

$$
g_{\left(\lambda_{0}+\varepsilon\right)}\left(\widetilde{\theta}_{\lambda_{n}}\right) \leq g_{\lambda_{n}}\left(\widetilde{\theta}_{\lambda_{n}}\right) \leq t
$$

Since $g_{\left(\lambda_{0}+\varepsilon\right)}$ is l.s.c. and a subsequence $\left(\widetilde{\theta}_{\psi(n)}\right)_{n \geq 0}$ of $\left(\widetilde{\theta}_{\lambda_{n}}\right)_{n \geq 0}$ converges towards $\widetilde{\theta}_{0}$, we obtain:

$$
g_{\left(\lambda_{0}+\varepsilon\right)}\left(\widetilde{\theta}_{0}\right) \leq \liminf _{n \rightarrow \infty} g_{\left(\lambda_{0}+\varepsilon\right)}\left(\widetilde{\theta}_{\psi(n)}\right) \leq t .
$$

This inequality is satisfied for all positive $\varepsilon$, and $\widetilde{\theta}_{0} \in \operatorname{ad}(\overline{\mathcal{D}})$, so

$$
\widetilde{\mathcal{K}}_{\theta, h}\left(\lambda_{0}\right) \leq g_{\lambda_{0}}\left(\widetilde{\theta}_{0}\right)=\lim _{\varepsilon \rightarrow 0, \varepsilon>0} g_{\left(\lambda_{0}+\varepsilon\right)}\left(\widetilde{\theta}_{0}\right) \leq t .
$$

This ends the proof of Lemma 4.1.

We now study the continuity of $\mathcal{K}_{\theta, h}$ :

Lemma 4.2. The function $\widetilde{\mathcal{K}}_{\theta, h}$ is continuous on $\mathbb{R}^{+}$, except possibly at $\delta_{\theta, h}$ where it may be only right continuous.

The proof of the continuity of $\widetilde{\mathcal{K}}_{\theta, h}$ is quite long and technical, and this property of $\widetilde{\mathcal{K}}_{\theta, h}$ is not needed to prove the large deviation principle. However, as explained in Section 3.5 and below, the continuity of $\widetilde{\mathcal{K}}_{\theta, h}$ is a natural question to ask, so it seems to us important to give an answer to it.

Proof. We define

$$
\widetilde{\mathcal{K}}_{\theta, h}\left(\lambda^{+}\right)=\lim _{\varepsilon \rightarrow 0, \varepsilon>0} \widetilde{\mathcal{K}}_{\theta, h}(\lambda+\varepsilon) \quad \text { and } \quad \widetilde{\mathcal{K}}_{\theta, h}\left(\lambda^{-}\right)=\lim _{\varepsilon \rightarrow 0, \varepsilon>0} \widetilde{\mathcal{K}}_{\theta, h}(\lambda-\varepsilon) .
$$

First of all, we prove that $\widetilde{\mathcal{K}}_{\theta, h}$ is right continuous, i.e., $\widetilde{\mathcal{K}}_{\theta, h}\left(\lambda^{+}\right)=\widetilde{\mathcal{K}}_{\theta, h}(\lambda)$ for all $\lambda \in \mathbb{R}^{+}$. We have for all $\lambda \geq 0$

$$
\begin{aligned}
\widetilde{\mathcal{K}}_{\theta, h}\left(\lambda^{+}\right) & =\lim _{\varepsilon \rightarrow 0, \varepsilon>0} \inf _{\tilde{\theta} \in \operatorname{ad}(\overline{\mathcal{D}})} \frac{1}{\cos (\widetilde{\theta}-\theta)} \mathcal{I}_{\widetilde{\theta}}\left((\lambda+\varepsilon) \cos (\widetilde{\theta}-\theta)^{+}\right) \\
& \leq \lim _{\varepsilon \rightarrow 0, \varepsilon>0} \inf _{\tilde{\theta} \in \operatorname{ad}(\overline{\mathcal{D}})} \frac{1}{\cos (\widetilde{\theta}-\theta)} \mathcal{I}_{\widetilde{\theta}}\left(\lambda \cos (\widetilde{\theta}-\theta)^{+}\right) \\
& \leq \widetilde{\mathcal{K}}_{\theta, h}(\lambda),
\end{aligned}
$$

and since $\widetilde{\mathcal{K}}_{\theta, h}$ is lower semi-continuous, we know that

$$
\widetilde{\mathcal{K}}_{\theta, h}\left(\lambda^{+}\right) \geq \widetilde{\mathcal{K}}_{\theta, h}(\lambda)
$$

thus

$$
\forall \lambda \geq 0 \quad \widetilde{\mathcal{K}}_{\theta, h}\left(\lambda^{+}\right)=\widetilde{\mathcal{K}}_{\theta, h}(\lambda) .
$$


On the other hand, for all $\lambda \geq 0$ we have

$$
\begin{aligned}
\widetilde{\mathcal{K}}_{\theta, h}\left(\lambda^{-}\right) & =\lim _{\varepsilon \rightarrow 0, \varepsilon>0} \inf _{\widetilde{\theta} \in \operatorname{ad}(\overline{\mathcal{D}})} \frac{1}{\cos (\widetilde{\theta}-\theta)} \mathcal{I}_{\widetilde{\theta}}\left((\lambda-\varepsilon) \cos (\widetilde{\theta}-\theta)^{+}\right) \\
& =\inf _{\widetilde{\theta} \in \operatorname{ad}(\overline{\mathcal{D}})} \lim _{\varepsilon \rightarrow 0, \varepsilon>0} \frac{1}{\cos (\widetilde{\theta}-\theta)} \mathcal{I}_{\widetilde{\theta}}\left((\lambda-\varepsilon) \cos (\widetilde{\theta}-\theta)^{+}\right) \\
& =\inf _{\tilde{\theta} \in \operatorname{ad}(\overline{\mathcal{D}})} \frac{1}{\cos (\widetilde{\theta}-\theta)} \mathcal{I}_{\widetilde{\theta}}\left(\lambda \cos (\widetilde{\theta}-\theta)^{-}\right) \\
& \geq \widetilde{\mathcal{K}}_{\theta, h}(\lambda)
\end{aligned}
$$

since the limit in $\varepsilon$ appearing in these equations is a decreasing limit. Thus $\widetilde{\mathcal{K}}_{\theta, h}$ is continuous at $\lambda$ if and only if

$$
\inf _{\widetilde{\theta} \in \operatorname{ad}(\overline{\mathcal{D}})} \frac{1}{\cos (\widetilde{\theta}-\theta)} \mathcal{I}_{\widetilde{\theta}}\left(\lambda \cos (\widetilde{\theta}-\theta)^{-}\right)=\inf _{\widetilde{\theta} \in \operatorname{ad}(\overline{\mathcal{D}})} \frac{1}{\cos (\widetilde{\theta}-\theta)} \mathcal{I}_{\widetilde{\theta}}\left(\lambda \cos (\widetilde{\theta}-\theta)^{+}\right) .
$$

We will thus prove that it is true for all $\lambda \neq \delta_{\theta, h}$. Notice that the proof we propose can be performed with $\operatorname{ad}(\underline{\mathcal{D}})$ instead of $\operatorname{ad}(\overline{\mathcal{D}})$, and thus the continuity of $\widetilde{\mathcal{K}}_{\theta, h}$ is linked with the existence of the limit

$$
\lim _{n \rightarrow \infty} \frac{1}{n l(A)} \log \mathbb{P}\left[\phi_{n} \leq \lambda n l(A)\right]
$$

as explained in Section 3.5. We need two intermediate lemmas to prove Lemma 4.2. The first one is the following:

Lemma 4.3. Let $\Lambda$ be the function defined on $\mathbb{R}^{+} \times \mathbb{R}^{2} \backslash(0,0)$ by

$$
\Lambda(\lambda, \vec{v})=\|\vec{v}\|_{2} \mathcal{I}_{\theta(\vec{v})}\left(\lambda(|\cos (\theta(\vec{v}))|+|\sin (\theta(\vec{v}))|)^{+}\right)
$$

where $\theta(\vec{v}) \in\left[0,2 \pi\left[\right.\right.$ satisfies $\vec{v}=\|\vec{v}\|_{2}(\cos (\theta(\vec{v})), \sin (\theta(\vec{v})))$ and $\|\vec{v}\|_{2}$ is the Euclidean norm of $\vec{v}$. Then for all vectors $\vec{u}$ and $\vec{v}$ in $\left(\mathbb{R}^{+}\right)^{2} \backslash\{(0,0)\}$, we have

$$
\forall \lambda \in \mathbb{R}^{+} \quad \Lambda(\lambda, \vec{u}+\vec{v}) \leq \Lambda(\lambda, \vec{u})+\Lambda(\lambda, \vec{v}),
$$

Proof. Lemma 4.3 is a simple consequence of Lemma 3.1. We consider $\vec{u}$ and $\vec{v}$ in $\left(\mathbb{R}^{+}\right)^{2} \backslash\{(0,0)\}$, and define $\vec{w}=\vec{u}+\vec{v}$. We use the notations $\theta(\vec{u})=\theta_{a}, \theta(\vec{v})=\theta_{b}$ and $\theta(\vec{w})=\theta_{c}$. We consider the triangle $(a b c)$ of side $[b c]$ (resp. $[a b],[a c]$ ) orthogonal to $\vec{u}$ (resp. $\vec{w}, \vec{v})$ and of length $\|\vec{u}\|_{2}$ (resp. $\|\vec{w}\|_{2},\|\vec{v}\|_{2}$ ). It is indeed a triangle since $\vec{w}=\vec{u}+\vec{v}$. Moreover, since $\vec{u}$ and $\vec{v}$ are in $\left(\mathbb{R}^{+}\right)^{2} \backslash\{(0,0)\}$, we know that the angles $\widehat{c a b}$ and $\widehat{a b c}$ have values strictly smaller than $\pi / 2$. We consider $\lambda \in \mathbb{R}^{+}$, and

$$
\lambda^{\prime}=\lambda l(a b)\left(\cos \theta_{c}+\sin \theta_{c}\right)=\lambda\|\vec{w}\|_{1} .
$$

We can apply Lemma 3.1 in the triangle $(a b c)$ to obtain for all $\alpha \in[0,1]$

$$
\begin{aligned}
\Lambda(\lambda, \vec{u}+\vec{v}) & =l(a b) \mathcal{I}_{\theta_{c}}\left({\frac{\lambda^{\prime}}{l(a b)}}^{+}\right) \\
& \leq l(a c) \mathcal{I}_{\theta_{b}}\left(\alpha{\frac{\lambda^{\prime}}{l(a c)}}^{+}\right)+l(b c) \mathcal{I}_{\theta_{a}}\left((1-\alpha){\frac{\lambda^{\prime}}{l(b c)}}^{+}\right) \\
& \leq \Lambda\left(\lambda \frac{\alpha\|\vec{w}\|_{1}}{\|\vec{v}\| 1}, \vec{v}\right)+\Lambda\left(\lambda \frac{(1-\alpha)\|\vec{w}\|_{1}}{\|\vec{u}\| 1}, \vec{u}\right) .
\end{aligned}
$$


Since $\vec{u}$ and $\vec{v}$ are both in $\left(\mathbb{R}^{+}\right)^{2}$, we know that $\|\vec{w}\|_{1}=\|\vec{u}\|_{1}+\|\vec{v}\|_{1}$, thus we can choose $\alpha=\|\vec{v}\|_{1} /\|\vec{w}\|_{1} \in[0,1]$ and $1-\alpha=\|\vec{u}\|_{1} /\|\vec{w}\|_{1}$. This ends the proof of Lemma 4.3 .

Lemma 4.3 states a property of convexity for the function $\Lambda$. To deduce from it a property of continuity, we need to investigate when $\Lambda$, thus $\mathcal{I}_{\tilde{\theta}}$, is finite. It is known (see Rossignol and Théret [14]) that $\mathcal{I}_{\tilde{\theta}}\left(\lambda^{+}\right)$is infinite if $\lambda \in[0, \delta(|\cos \widetilde{\theta}|+|\sin \widetilde{\theta}|)[($ if $\delta>0)$ and finite if $\lambda \in] \delta(|\cos \widetilde{\theta}|+|\sin \widetilde{\theta}|),+\infty$. The only point to study is the behaviour of $\mathcal{I}_{\widetilde{\theta}}\left(\delta(|\cos \widetilde{\theta}|+|\sin \widetilde{\theta}|)^{+}\right)$. This is the purpose of the following Lemma:

Lemma 4.4. We have

$$
\begin{gathered}
\forall \widetilde{\theta}, \mathcal{I}_{\widetilde{\theta}}\left(\delta(|\cos \widetilde{\theta}|+|\sin \widetilde{\theta}|)^{+}\right)<\infty \quad \Longleftrightarrow \quad \exists \widetilde{\theta}, \mathcal{I}_{\widetilde{\theta}}\left(\delta(|\cos \widetilde{\theta}|+|\sin \widetilde{\theta}|)^{+}\right)<\infty \\
\Longleftrightarrow \mathbb{P}(t(e)=\delta)>0,
\end{gathered}
$$

Proof. First, let us prove that

$$
\mathbb{P}(t(e)=\delta)>0 \Longrightarrow \forall \widetilde{\theta}, \mathcal{I}_{\widetilde{\theta}}\left(\delta(|\cos \widetilde{\theta}|+|\sin \widetilde{\theta}|)^{+}\right)<\infty
$$

Let $\widetilde{A}$ be a line segment orthogonal to $(\cos \widetilde{\theta}, \sin \widetilde{\theta})$ for some fixed $\widetilde{\theta}$, and $h$ a height function satisfying $\lim _{n \rightarrow \infty} h(n)=+\infty$. We know (see Lem. 4.1 in Rossignol and Théret [14]) that the minimal number of edges $\mathcal{N}(n \widetilde{A}, h(n))$ of a cutset that separates $(n \widetilde{A})_{1}^{h(n), 1 / 2, \widetilde{\theta}}$ from $(n \widetilde{A})_{2}^{h(n), 1 / 2, \widetilde{\theta}}$ satisfies

$$
\left|\frac{\mathcal{N}(n \widetilde{A}, h(n))}{n l(\widetilde{A})}-(|\cos \widetilde{\theta}|+|\sin \widetilde{\theta}|)\right| \leq \frac{2}{n l(\widetilde{A})} .
$$

Let $\varepsilon>0$. Let $E_{\min }(n)$ be a cutset of minimal number of edges. For $n$ large enough, we know that $1 / \sqrt{n} \leq \varepsilon / 2$ and $\mathcal{N}(n \widetilde{A}, h(n)) /(n l(\widetilde{A})) \leq(|\cos \widetilde{\theta}|+|\sin \widetilde{\theta}|)+\varepsilon /(2 \delta)$, and we obtain

$$
\begin{aligned}
\mathbb{P}\left(\frac{\tau(n \widetilde{A}, h(n))}{n l(\widetilde{A})}\right. & \left.\leq \delta(|\cos \widetilde{\theta}|+|\sin \widetilde{\theta}|)+\varepsilon-\frac{1}{\sqrt{n}}\right) \\
& \geq \mathbb{P}\left(\frac{V\left(E_{\min }(n)\right)}{n l(\widetilde{A})} \leq \delta(|\cos \widetilde{\theta}|+|\sin \widetilde{\theta}|)+\frac{\varepsilon}{2}\right) \\
& \geq \mathbb{P}\left(\forall e \in E_{\min }(n), t(e)=\delta\right) \\
& \geq \mathbb{P}(t(e)=\delta)^{\mathcal{N}(n \widetilde{A}, h(n))} .
\end{aligned}
$$

Thus for all $\varepsilon>0$ we have

$$
\mathcal{I}_{\widetilde{\theta}}(\delta(|\cos \widetilde{\theta}|+|\sin \widetilde{\theta}|)+\varepsilon) \leq-(|\cos \widetilde{\theta}|+|\sin \widetilde{\theta}|) \log \mathbb{P}(t(e)=\delta),
$$

and we conclude that

$$
\mathcal{I}_{\widetilde{\theta}}\left(\delta(|\cos \widetilde{\theta}|+|\sin \widetilde{\theta}|)^{+}\right) \leq-(|\cos \widetilde{\theta}|+|\sin \widetilde{\theta}|) \log \mathbb{P}(t(e)=\delta) .
$$

This implies (4.27). We now prove that if for all distribution function $G$ such that $\inf \{x \mid G(x)>0\}=0$, we have

$$
G(0)=0 \quad \Longrightarrow \quad \forall \widetilde{\theta}, \mathcal{I}_{\tilde{\theta}}^{[G]}\left(0^{+}\right)=+\infty
$$

where the exponent $[G]$ stress the dependence of $\mathcal{I}$ in $G$, then for all distribution function $F$ on $\mathbb{R}^{+}$, if $\delta=$ $\inf \{x \mid F(x)>0\}$, we obtain:

$$
F(\delta)=0 \quad \Longrightarrow \quad \forall \widetilde{\theta}, \mathcal{I}_{\widetilde{\theta}}^{[F]}\left(\delta(|\cos \widetilde{\theta}|+|\sin \widetilde{\theta}|)^{+}\right)=+\infty
$$


Let $F$ be a distribution function on $\mathbb{R}^{+}, \delta=\inf \{x \mid F(x)>0\}$, and $(t(e))$ the family of capacities on the edges of distribution function $F$. Let $t^{\prime}(e)=t(e)-\delta \geq 0$ for all $e, t^{\prime}(e)$ has distribution function $G$ such that $\inf \{x \mid G(x)>0\}=0$, and $G(0)=F(\delta)$. We denote by $\tau$ (resp. $\tau^{\prime}$ ) the maximal flows corresponding to the capacities $(t(e))$ (resp. $\left.\left(t^{\prime}(e)\right)\right)$. Then obviously

$$
\tau(n \widetilde{A}, h(n)) \geq \tau^{\prime}(n \widetilde{A}, h(n))+\delta \mathcal{N}(n \widetilde{A}, h(n))
$$

thus for $n$ large enough, thanks to (4.28), we have

$$
\mathbb{P}\left(\frac{\tau(n \widetilde{A}, h(n))}{n l(\widetilde{A})} \leq \delta(|\cos \widetilde{\theta}|+|\sin \widetilde{\theta}|)+\varepsilon-\frac{1}{\sqrt{n}}\right) \leq \mathbb{P}\left(\frac{\tau^{\prime}(n \widetilde{A}, h(n))}{n l(\widetilde{A})} \leq \frac{\varepsilon}{4}\right)
$$

Thus

$$
\mathcal{I}_{\widetilde{\theta}}^{[F]}\left(\delta(|\cos \widetilde{\theta}|+|\sin \widetilde{\theta}|)^{+}\right) \geq \mathcal{I}_{\widetilde{\theta}}^{[G]}\left(0^{+}\right),
$$

which proves the previous statement. The last thing to prove is that if $F$ is a distribution function such that $\inf \{x \mid F(x)>0\}=0$, then

$$
F(0)=\mathbb{P}(t(e)=0)=0 \quad \Longrightarrow \quad \forall \widetilde{\theta}, \mathcal{I}_{\widetilde{\theta}}\left(0^{+}\right)=+\infty
$$

We consider such a distribution function $F$. We want to compare $F$ with a Bernoulli distribution of parameter $p$ very close to 1 . For a fixed $p$ (as close to 1 as we will need), there exists $\eta(p)>0$ such that $F(\eta(p))<1-p$, because $F(0)=0$ and $F$ is right continuous. We denote by $\left(t^{[p]}(e)\right)$ the i.i.d. family of Bernoulli variables of parameter $p$ indexed by the edges, and by $\tau^{[p]}$ the maximal flow corresponding to these capacities. Then

$$
\tau(n \widetilde{A}, h(n)) \geq \eta(p) \tau^{[p]}(n \widetilde{A}, h(n)) .
$$

It is proved in Section 3 of Théret [16] (the proof is written for a straight cylinder $\tilde{\theta}=0$ and for the variable $\phi$, but it can be directly adapted to a tilted box and the variable $\tau$ - notice that the factor $h(n)$ disappears) that there exists a constant $c$ such that for all $\gamma>0$

$$
\mathbb{P}\left(\frac{\tau^{[p]}(n \widetilde{A}, h(n))}{n l(\widetilde{A})} \leq \frac{1}{2}\right) \leq \exp \left(-n l(\widetilde{A})\left[\frac{\gamma}{2}-\log c-\log \left(p+(1-p) \mathrm{e}^{\gamma}\right)\right]\right)
$$

Thus for any fixed $R$ (very large, thus $\log c<R$ ), we can choose $\gamma=6 R$, and then $p(R)$ close enough to 1 to obtain that $\log \left(p+(1-p) \mathrm{e}^{6 R}\right)<R$, thus

$$
\mathbb{P}\left(\frac{\tau^{[p(R)]}(n \widetilde{A}, h(n))}{n l(\widetilde{A})} \leq \frac{1}{2}\right) \leq \mathrm{e}^{-\operatorname{Rnl}(\widetilde{A})}
$$

Finally, for any fixed $R$, for a fixed $\varepsilon$ small enough to have $\varepsilon / \eta(p(R)) \leq 1 / 2$, we obtain

$$
\mathbb{P}\left(\frac{\tau(n \widetilde{A}, h(n))}{n l(\widetilde{A})} \leq \varepsilon\right) \leq \mathbb{P}\left(\frac{\tau^{[p(R)]}(n \widetilde{A}, h(n))}{n l(\widetilde{A})} \leq \frac{\varepsilon}{\eta(p(R))}\right) \leq \mathrm{e}^{-R n l(\widetilde{A})},
$$

thus $\mathcal{I}_{\widetilde{\theta}}(\varepsilon) \geq R$ for such small $\varepsilon$, which implies that $\mathcal{I}_{\widetilde{\theta}}\left(0^{+}\right) \geq R$ for all $R$. This ends the proof of equation (4.29), and thus the proof of Lemma 4.4 . 
We come back to the proof of Lemma 4.2. We recall that $\widetilde{\mathcal{K}}_{\theta, h}(\lambda)=\inf \left\{g_{\lambda}(\widetilde{\theta}) \mid \widetilde{\theta} \in \operatorname{ad}(\overline{\mathcal{D}})\right\}$. Since $g_{\lambda}$ is 1.s.c. and $\operatorname{ad}(\overline{\mathcal{D}})$ is compact, there exists $\widetilde{\theta}_{\lambda} \in \operatorname{ad}(\overline{\mathcal{D}})$ (maybe not unique) such that $\widetilde{\mathcal{K}}_{\theta, h}(\lambda)=g_{\lambda}\left(\widetilde{\theta}_{\lambda}\right)$. If

$$
\lambda \cos \left(\widetilde{\theta}_{\lambda}-\theta\right)<\delta\left(\left|\cos \widetilde{\theta}_{\lambda}\right|+\left|\sin \widetilde{\theta}_{\lambda}\right|\right),
$$

then

If

$$
\widetilde{\mathcal{K}}_{\theta, h}(\lambda)=\mathcal{I}_{\widetilde{\theta}_{\lambda}}\left(\lambda \cos \left(\widetilde{\theta}_{\lambda}-\theta\right)^{+}\right)=+\infty=\widetilde{\mathcal{K}}_{\theta, h}\left(\lambda^{-}\right) .
$$

then

$$
\lambda \cos \left(\widetilde{\theta}_{\lambda}-\theta\right)>\delta\left(\left|\cos \widetilde{\theta}_{\lambda}\right|+\left|\sin \widetilde{\theta}_{\lambda}\right|\right),
$$

$$
\begin{aligned}
\widetilde{\mathcal{K}}_{\theta, h}\left(\lambda^{-}\right) & =\lim _{\varepsilon \rightarrow 0, \varepsilon>0} \widetilde{\mathcal{K}}_{\theta, h}(\lambda-\varepsilon) \\
& \leq \lim _{\varepsilon \rightarrow 0, \varepsilon>0} \frac{1}{\cos \left(\widetilde{\theta}_{\lambda}-\theta\right)} \mathcal{I}_{\widetilde{\theta}_{\lambda}}\left((\lambda-\varepsilon) \cos \left(\widetilde{\theta}_{\lambda}-\theta\right)^{+}\right) \\
& \leq \frac{1}{\cos \left(\widetilde{\theta}_{\lambda}-\theta\right)} \mathcal{I}_{\widetilde{\theta}_{\lambda}}\left(\lambda \cos \left(\widetilde{\theta}_{\lambda}-\theta\right)^{-}\right) \\
& \leq \frac{1}{\cos \left(\widetilde{\theta}_{\lambda}-\theta\right)} \mathcal{I}_{\widetilde{\theta}_{\lambda}}\left(\lambda \cos \left(\widetilde{\theta}_{\lambda}-\theta\right)^{+}\right)=\widetilde{\mathcal{K}}_{\theta, h}(\lambda),
\end{aligned}
$$

since $\mathcal{I}_{\tilde{\theta}}$ is continuous on $] \delta(|\cos \widetilde{\theta}|+|\sin \widetilde{\theta}|),+\infty\left[\right.$, thus $\widetilde{\mathcal{K}}_{\theta, h}\left(\lambda^{-}\right)=\widetilde{\mathcal{K}}_{\theta, h}(\lambda)$. We suppose that

$$
\lambda \cos \left(\widetilde{\theta}_{\lambda}-\theta\right)=\delta\left(\left|\cos \widetilde{\theta}_{\lambda}\right|+|\sin \widetilde{\theta}|_{\lambda}\right),
$$

which is the only non-obvious case, and thus

$$
\widetilde{\mathcal{K}}_{\theta, h}(\lambda)=\frac{1}{\cos \left(\widetilde{\theta}_{\lambda}-\theta\right)} \mathcal{I}_{\widetilde{\theta}_{\lambda}}\left(\delta\left(\left|\cos \widetilde{\theta}_{\lambda}\right|+\left|\sin \widetilde{\theta}_{\lambda}\right|\right)^{+}\right) .
$$

If $\mathbb{P}(t(e)=\delta)=0$, by Lemma 4.4 we know that the previous quantity is infinite, thus $\widetilde{\mathcal{K}}_{\theta, h}\left(\lambda^{-}\right)=+\infty=\widetilde{\mathcal{K}}_{\theta, h}(\lambda)$. For the rest of the proof, we suppose that $\mathbb{P}(t(e)=\delta)>0$. Still by Lemma 4.4, we know in this case that for all $\widetilde{\theta}, \mathcal{I}_{\widetilde{\theta}}\left(\delta(|\cos \widetilde{\theta}|+|\sin \widetilde{\theta}|)^{+}\right)<+\infty$. If $\lambda=\delta_{\theta, h}$, we have nothing to prove, thus we suppose that $\lambda>\delta_{\theta, h}$ (it implies that $\lambda>0)$. We suppose that the following property $\mathcal{P}$ holds: there exists a sequence $\left(\widetilde{\theta}_{n}\right)_{n \in \mathbb{N}}$ such that

$$
\mathcal{P}\left\{\begin{array}{l}
\text { (i) } \lim _{n \rightarrow \infty} \widetilde{\theta}_{n}=\widetilde{\theta}_{\lambda}, \\
\text { (ii) } \forall n \in \mathbb{N}, \delta \frac{\left|\cos \widetilde{\theta}_{n}\right|+\left|\sin \tilde{\theta}_{n}\right|}{\cos \left(\tilde{\theta}_{n}-\theta\right)}<\lambda=\delta \frac{\left|\cos \tilde{\theta}_{\lambda}\right|+\left|\sin \tilde{\theta}_{\lambda}\right|}{\cos \left(\tilde{\theta}_{\lambda}-\theta\right)}, \\
\text { (iii) } \lim \sup _{n \rightarrow \infty} \mathcal{I}_{\widetilde{\theta}_{n}}\left(\delta\left(\left|\cos \widetilde{\theta}_{n}\right|+\left|\sin \widetilde{\theta}_{n}\right|\right)^{+}\right) \leq \mathcal{I}_{\widetilde{\theta}_{\lambda}}\left(\delta\left(\left|\cos \widetilde{\theta}_{\lambda}\right|+\left|\sin \widetilde{\theta}_{\lambda}\right|\right)^{+}\right),
\end{array}\right.
$$

We consider a given $\eta>0$. For $n_{0}$ large enough, we have

$$
\frac{1}{\cos \left(\widetilde{\theta}_{n_{0}}-\theta\right)} \mathcal{I}_{\widetilde{\theta}_{n_{0}}}\left(\delta\left(\left|\cos \widetilde{\theta}_{n_{0}}\right|+\left|\sin \widetilde{\theta}_{n_{0}}\right|\right)^{+}\right) \leq \frac{1}{\cos \left(\widetilde{\theta}_{\lambda}-\theta\right)} \mathcal{I}_{\widetilde{\theta}_{\lambda}}\left(\delta\left(\left|\cos \widetilde{\theta}_{\lambda}\right|+\left|\sin \widetilde{\theta}_{\lambda}\right|\right)^{+}\right)+\eta
$$

Moreover, there exists $\varepsilon_{0}>0$ such that

$$
\delta \frac{\left|\cos \widetilde{\theta}_{n_{0}}\right|+\left|\sin \widetilde{\theta}_{n_{0}}\right|}{\cos \left(\widetilde{\theta}_{n_{0}}-\theta\right)} \leq \lambda-\varepsilon_{0}
$$


and for all $\varepsilon \leq \varepsilon_{0}$, since $\mathcal{I}_{\widetilde{\theta}_{n_{0}}}$ is non increasing, we obtain that

$$
\begin{aligned}
\widetilde{\mathcal{K}}_{\theta, h}(\lambda-\varepsilon) & \leq \frac{1}{\cos \left(\widetilde{\theta}_{n_{0}}-\theta\right)} \mathcal{I}_{\widetilde{\theta}_{n_{0}}}\left((\lambda-\varepsilon) \cos \left(\widetilde{\theta}_{n_{0}}-\theta\right)^{+}\right) \\
& \leq \frac{1}{\cos \left(\widetilde{\theta}_{n_{0}}-\theta\right)} \mathcal{I}_{\widetilde{\theta}_{n_{0}}}\left(\delta\left(\left|\cos \widetilde{\theta}_{n_{0}}\right|+\left|\sin \widetilde{\theta}_{n_{0}}\right|\right)^{+}\right) \\
& \leq \frac{1}{\cos \left(\widetilde{\theta}_{\lambda}-\theta\right)} \mathcal{I}_{\widetilde{\theta}_{\lambda}}\left(\delta\left(\left|\cos \widetilde{\theta}_{\lambda}\right|+\left|\sin \widetilde{\theta}_{\lambda}\right|\right)^{+}\right)+\eta=\widetilde{\mathcal{K}}_{\theta, h}(\lambda)+\eta .
\end{aligned}
$$

We conclude that $\widetilde{\mathcal{K}}_{\theta, h}\left(\lambda^{-}\right) \leq \widetilde{\mathcal{K}}_{\theta, h}(\lambda)$, so $\widetilde{\mathcal{K}}_{\theta, h}\left(\lambda^{-}\right)=\widetilde{\mathcal{K}}_{\theta, h}(\lambda)$, and this ends the proof of Lemma 4.2 .

The last thing we have to do is to prove the property $\mathcal{P}$. Obviously, property (ii) is linked with the monotonicity of the function

$$
\Gamma: \widetilde{\theta} \mapsto \frac{|\cos \widetilde{\theta}|+|\sin \widetilde{\theta}|}{\cos (\widetilde{\theta}-\theta)} .
$$

If $\theta \in\{k \pi / 4 \mid k \in \mathbb{N}\}$, we will prove in the next paragraph, see Lemma 4.5 , that $\widetilde{\mathcal{K}}_{\theta, h}(\lambda)=\mathcal{I}_{\theta}\left(\lambda^{+}\right)$; since it is obvious that in this case $\delta_{\theta, h}=\delta$, the continuity of $\widetilde{\mathcal{K}}_{\theta, h}$ possibly except at $\delta_{\theta, h}$ is already known. We suppose that $\theta \notin\{k \pi / 4 \mid k \in \mathbb{N}\}$, and by symmetry we can suppose that $\theta \in] 0, \pi / 2[\backslash\{\pi / 4\}$. It is obvious (see the factor $\left.\cos (\widetilde{\theta}-\theta)^{-1}\right)$ that for all $\widetilde{\theta} \in[\theta-\pi / 2, \theta+\pi / 2] \backslash[0, \pi / 2]$, we have $\Gamma(\widetilde{\theta})>\inf _{[0, \pi / 2]} \Gamma$, so $\operatorname{argmin} \Gamma \in[0, \pi / 2]$. Similarly, $\widetilde{\theta}_{\lambda} \in[0, \pi / 2]$ too. For all $\widetilde{\theta} \in[0, \pi / 2]$, we can write

$$
\Gamma(\widetilde{\theta})=\frac{\cos \widetilde{\theta}+\sin \widetilde{\theta}}{\cos (\widetilde{\theta}-\theta)}=\frac{1+\tan \widetilde{\theta}}{\cos \theta+\sin \theta \tan \widetilde{\theta}} .
$$

We deduce from this equality that $\Gamma$ is strictly monotone on $[0, \pi / 2]$ : strictly increasing (resp. decreasing) if $\theta \in] 0, \pi / 4[$ (resp. $\theta \in] \pi / 4, \pi / 2[$ ), and thus $\operatorname{argmin} \Gamma=0$ (resp. $\operatorname{argmin} \Gamma=\pi / 2)$. We consider the case $\theta \in] 0, \pi / 4[$, the study of the case $\theta \in] \pi / 4, \pi / 2\left[\right.$ being similar. We know that $\left.\left.\widetilde{\theta}_{\lambda} \in\right] 0, \pi / 2\right]$, because $\widetilde{\theta}_{\lambda}=0$ implies that $\lambda=\delta_{\theta, h}$, and we excluded this case. Thus we can consider a strictly increasing sequence $\left(\widetilde{\theta}_{n}\right)_{n \in \mathbb{N}}$ such that $\left.\widetilde{\theta}_{n} \in\right] 0, \pi / 2\left[\right.$ for all $n$ and (i): $\lim _{n \rightarrow \infty} \widetilde{\theta}_{n}=\widetilde{\theta}_{\lambda}$ is satisfied. Since $\Gamma$ is strictly increasing on $[0, \pi / 2]$, we know that such a strictly increasing sequence $\left(\widetilde{\theta}_{n}\right)_{n \in \mathbb{N}}$ satisfies the hypothesis (ii). To prove that (iii) also holds, we need Lemmas 4.3 and 4.4. The function

$$
\Lambda_{\delta}: \vec{v} \mapsto \Lambda(\delta, \vec{v})
$$

is finite on $\left(\mathbb{R}^{+}\right)^{2} \backslash\{(0,0)\}$ under the hypothesis that $F(\delta)>0$ we did (see Lem. 4.4), and it is convex (see Lemma 4.3), so it is continuous on the interior of $\left(\mathbb{R}^{+}\right)^{2} \backslash\{(0,0)\}$. If $\widetilde{\theta}_{\lambda} \neq \pi / 2$, it proves (iii). We suppose $\widetilde{\theta}_{\lambda}=\pi / 2$. Let $\vec{u}=(0,1), \vec{v}_{n}=\left(1 / \tan \widetilde{\theta}_{n}, 0\right)$ and $\vec{w}_{n}=\left(1 / \tan \widetilde{\theta}_{n}, 1\right)=\vec{u}+\vec{v}_{n}$. By equation $(4.26)$ for $\lambda=\delta$ we have for all $n \in \mathbb{N}$

$$
\frac{1}{\sin \widetilde{\theta}_{n}} \mathcal{I}_{\widetilde{\theta}_{n}}\left(\delta\left(\left|\cos \widetilde{\theta}_{n}\right|+\left|\sin \widetilde{\theta}_{n}\right|\right)^{+}\right) \leq \mathcal{I}_{\pi / 2}\left(\delta^{+}\right)+\frac{1}{\tan \widetilde{\theta}_{n}} \mathcal{I}_{0}\left(\delta^{+}\right)
$$

and sending $n$ to infinity we obtain (iii), so the property $\mathcal{P}$ is proved.

We prove finally the property stated in Remark 2.10 , in fact a property a little bit more general:

Lemma 4.5. If $\theta \in\{k \pi / 4 \mid k \in \mathbb{N}\}$, then

$$
\mathcal{K}_{\theta, h}=\mathcal{J}_{\theta} .
$$

Proof. We fix a $\theta \in\{k \pi / 4 \mid k \in \mathbb{N}\}$. We know that $\nu_{\theta}=\eta_{\theta, h}$ for such a $\theta$ (see Rem. 2.11 in Rossignol and Théret [13]), so it is sufficient to prove that

$$
\forall \lambda \geq 0, \quad \widetilde{\mathcal{K}}_{\theta, h}(\lambda)=\mathcal{I}_{\theta}\left(\lambda^{+}\right) .
$$


Since $\theta \in \operatorname{ad}(\overline{\mathcal{D}})$, it is equivalent to prove that

$$
\forall \lambda \geq 0, \forall \widetilde{\theta} \in[\theta-\pi / 2, \theta+\pi / 2], \quad \mathcal{I}_{\theta}\left(\lambda^{+}\right) \leq \frac{1}{\cos (\widetilde{\theta}-\theta)} \mathcal{I}_{\tilde{\theta}}\left(\lambda \cos (\widetilde{\theta}-\theta)^{+}\right) .
$$

Let $\tilde{\theta} \in] \theta-\pi / 2, \theta+\pi / 2[$. We use the same notations as in Lemma 3.1. We consider the non degenerate triangle $(a b c)$ such that $\theta_{c}=\theta+\pi\left(\operatorname{socyl}_{c}(n)\right.$ is a straight cylinder in the case $\left.\theta=0\right), \theta_{b}=\max (\widetilde{\theta}, 2 \theta-\widetilde{\theta})$, $\theta_{a}=\min (\widetilde{\theta}, 2 \theta-\widetilde{\theta}), l(a b)=1$ and $l(a c)=l(b c)=(2 \cos (\widetilde{\theta}-\theta))^{-1}$. Since the graph is invariant by a symmetry of axis $((0,0),(\cos \theta, \sin \theta))$ (respectively $((0,0),(1,1)))$, we know that $\mathcal{I}_{\theta_{a}}=\mathcal{I}_{\theta_{b}}$ (respectively $\left.\mathcal{I}_{\theta_{c}}=\mathcal{I}_{\theta}\right)$. Then Lemma 3.1 applied with $\alpha=1 / 2$ states that for all $\lambda \geq 0$,

$$
\mathcal{I}_{\theta}\left(\lambda^{+}\right) \leq \frac{1}{\cos (\widetilde{\theta}-\theta)} \mathcal{I}_{\widetilde{\theta}}\left(\lambda \cos (\widetilde{\theta}-\theta)^{+}\right)
$$

The inequality remains obviously valid for $\widetilde{\theta} \in\{\theta+\pi / 2, \theta-\pi / 2\}$, since we have seen in Remark 3.3 that the right hand side of the previous inequality equals $+\infty$ in this case. This ends the proof of Lemma 4.5 .

\subsection{Lower bound}

We have to prove that for all open subset $\mathcal{O}$ of $\mathbb{R}^{+}$, we have

$$
\liminf _{n \rightarrow \infty} \frac{1}{n l(A)} \log \mathbb{P}\left[\frac{\phi_{n}}{n l(A)} \in \mathcal{O}\right] \geq-\inf _{\lambda \in \mathcal{O}} \mathcal{K}_{\theta, h}(\lambda) .
$$

We will use standard arguments, see for example Cerf [3] Section 8.1. Classically, it suffices to prove the local lower bound:

$$
\forall a \in \mathbb{R}^{+}, \forall \varepsilon>0 \quad \liminf _{n \rightarrow \infty} \frac{1}{n l(A)} \log \mathbb{P}\left[\frac{\phi_{n}}{n l(A)} \in[a-\varepsilon, a+\varepsilon]\right] \geq-\mathcal{K}_{\theta, h}(a),
$$

If $\mathcal{K}_{\theta, h}(a)=+\infty$, the result is obvious, so we suppose that $\mathcal{K}_{\theta, h}(a)<+\infty$. For all $\eta<\varepsilon$, we have

$$
\begin{aligned}
\liminf _{n \rightarrow \infty} \frac{1}{n l(A)} \log \mathbb{P} & {\left[\frac{\phi_{n}}{n l(A)} \in[a-\varepsilon, a+\varepsilon]\right] } \\
& \geq \liminf _{n \rightarrow \infty} \frac{1}{n l(A)} \log \left(\mathbb{P}\left[\frac{\phi_{n}}{n l(A)} \leq a+\eta\right]-\mathbb{P}\left[\frac{\phi_{n}}{n l(A)} \leq a-\varepsilon\right]\right) .
\end{aligned}
$$

Since $\mathcal{K}_{\theta, h}$ is strictly decreasing (see Lem. 3.4), we deduce that for all $a \in \mathbb{R}^{+}$such that $\mathcal{K}_{\theta, h}(a)<\infty$, for all positive $\eta$ and $\varepsilon$, we have

$$
\inf _{\tilde{\theta} \in \mathcal{D}} \frac{1}{\cos (\widetilde{\theta}-\theta)} \mathcal{I}_{\widetilde{\theta}}\left((a+\eta) \cos (\widetilde{\theta}-\theta)^{-}\right)<\inf _{\tilde{\theta} \in \mathcal{D}} \frac{1}{\cos (\widetilde{\theta}-\theta)} \mathcal{I}_{\widetilde{\theta}}\left((a-\varepsilon) \cos (\widetilde{\theta}-\theta)^{+}\right) .
$$

Indeed, for all positive $\eta$, we have

$$
\inf _{\tilde{\theta} \in \mathcal{D}} \frac{1}{\cos (\widetilde{\theta}-\theta)} \mathcal{I}_{\widetilde{\theta}}\left((a+\eta) \cos (\widetilde{\theta}-\theta)^{-}\right) \leq \mathcal{K}_{\theta, h}(a)<\mathcal{K}_{\theta, h}(a-\varepsilon) .
$$


Then thanks to (3.10), (3.18) and (4.32), we know that the second term in the sum appearing in (4.31) is negligible compared to the first one, so we obtain that

$$
\begin{aligned}
\liminf _{n \rightarrow \infty} \frac{1}{n l(A)} \log \mathbb{P} & {\left[\frac{\phi_{n}}{n l(A)} \in[a-\varepsilon, a+\varepsilon]\right] } \\
& \geq-\inf _{\widetilde{\theta} \in \mathcal{D}} \frac{1}{\cos (\widetilde{\theta}-\theta)} \mathcal{I}_{\widetilde{\theta}}\left((a+\eta) \cos (\widetilde{\theta}-\theta)^{-}\right) \\
& \geq-\inf _{\widetilde{\theta} \in \mathcal{D}} \lim _{\varepsilon^{\prime} \rightarrow 0} \frac{1}{\cos (\widetilde{\theta}-\theta)} \mathcal{I}_{\widetilde{\theta}}\left((a+\eta) \cos (\widetilde{\theta}-\theta)-\varepsilon^{\prime}\right)
\end{aligned}
$$

Sending $\eta$ to zero, we obtain that

$$
\begin{aligned}
\liminf _{n \rightarrow \infty} \frac{1}{n l(A)} \log \mathbb{P}\left[\frac{\phi_{n}}{n l(A)} \in[a-\varepsilon, a+\varepsilon]\right] & \geq-\liminf _{\eta \rightarrow 0} \inf _{\widetilde{\theta} \in \mathcal{D}} \lim _{\varepsilon^{\prime} \rightarrow 0} \frac{1}{\cos (\widetilde{\theta}-\theta)} \mathcal{I}_{\widetilde{\theta}}\left((a+\eta) \cos (\widetilde{\theta}-\theta)-\varepsilon^{\prime}\right) \\
& \geq-\inf _{\widetilde{\theta} \in \mathcal{D}} \lim _{\eta \rightarrow 0} \lim _{\varepsilon^{\prime} \rightarrow 0} \frac{1}{\cos (\widetilde{\theta}-\theta)} \mathcal{I}_{\widetilde{\theta}}\left((a+\eta) \cos (\widetilde{\theta}-\theta)-\varepsilon^{\prime}\right) \\
& \geq-\inf _{\widetilde{\theta} \in \mathcal{D} \cos (\widetilde{\theta}-\theta)} \frac{1}{\mathcal{I}_{\widetilde{\theta}}\left(a \cos (\widetilde{\theta}-\theta)^{+}\right)}
\end{aligned}
$$

and so the local lower bound is proved.

\subsection{Upper bound}

\subsubsection{Upper large deviations}

To handle the upper large deviations, we shall use the following result:

Lemma 4.6. Let $A$ be a non-empty line-segment in $\mathbb{R}^{2}$, with Euclidean length $l(A)$. Let $\theta \in[0, \pi[$ be such that $(\cos \theta, \sin \theta)$ is orthogonal to the hyperplane spanned by $A$ and $h: \mathbb{N} \rightarrow \mathbb{R}^{+}$. We suppose that $(\mathbf{H 1}),(\mathbf{H 2}),(\mathbf{F 2})$, (FH1) and either $(\mathbf{F 4})$ or $(\mathbf{H 3})$ hold. Then for all $\lambda>\eta_{\theta, h}$ we have

$$
\limsup _{n \rightarrow \infty} \frac{1}{n l(A)} \log \mathbb{P}\left[\frac{\phi_{n}}{n l(A)} \geq \lambda\right]=-\infty .
$$

In fact, we have a stronger result, if $F$ admits an exponential moment:

Lemma 4.7. Let $A$ be a non-empty line-segment in $\mathbb{R}^{2}$, with Euclidean length $l(A)$. Let $\theta \in[0, \pi[$ be such that $(\cos \theta, \sin \theta)$ is orthogonal to the hyperplane spanned by $A$ and $h: \mathbb{N} \rightarrow \mathbb{R}^{+}$. We suppose that (H1), (H2), (F2), (FH1) and (F4) hold. Then for all $\lambda>\eta_{\theta, h}$, we have

$$
\liminf _{n \rightarrow \infty} \frac{-1}{n l(A) h(n)} \log \mathbb{P}[\phi(n A, h(n)) \geq \lambda n l(A)]>0 .
$$

The upper large deviations are thus of volume order.

Obviously, Lemma 4.7 implies Lemma 4.6 in the case where the condition (F4) is satisfied, since $\lim _{n \rightarrow \infty} h(n)=+\infty$ by $(\mathbf{H 1})$. We do not present a complete version of the proof of Lemma 4.7: it is simply a modification of the proofs of Theorem 2 in Théret [15] (the part concerning the positivity of the rate function, Sect. 3.7) and Theorem 4 in Théret [18], and it can be found in Part 3, Chapter 6, Section 5 of Théret [17]. The common idea of these proofs is the following. We consider the cylinder cyl $(N A, h(N))$, and divide it into slabs of height $2 h(n)$, i.e., translates of $\operatorname{cyl}(N A, h(n))$, for $n$ a lot smaller than $N$. If $\phi(N A, h(N))$ 
is big, it implies that the maximal flow from the top to the bottom of each slab is big too, and we have of order $h(N)$ such slabs for a fixed $n$. It implies roughly that

$$
\mathbb{P}[\phi(N A, h(N)) \geq \lambda N l(A)] “ \leq " \mathbb{P}[\phi(N A, h(n)) \geq \lambda N l(A)]^{p h(N)},
$$

for some constant $p$. We divide then each slab into disjoint translates of $\operatorname{cyl}(n A, h(n))$, and we can compare the maximal flow from the top to the bottom of the slab with the sum of the variables $\tau_{n}^{i}$ into these small cylinders. Roughly speaking, we obtain that

$$
\mathbb{P}[\phi(N A, h(n)) \geq \lambda N l(A)] " \leq " \mathbb{P}\left[\sum_{i} \tau_{n}^{i} \geq \lambda N l(A)\right] .
$$

Under the hypothesis (F4), Cramér's Theorem in $\mathbb{R}$ states that $\mathbb{P}\left[\sum_{i} \tau_{n}^{i} \geq \lambda N l(A)\right]$ decays exponentially fast with $N$ for any $\lambda>\nu_{\theta}=\lim _{n \rightarrow \infty} \tau_{n}^{i} /(n l(A))$, thus we obtain that

$$
\mathbb{P}[\phi(N A, h(N)) \geq \lambda N l(A)] \leq p^{\prime} \mathrm{e}^{-p^{\prime \prime} h(N) N l(A)},
$$

for other constants $p^{\prime}$ and $p^{\prime \prime}$. The only adaptation we have to do is to take into account the fact that under hypothesis (FH1), the limit $\eta_{\theta, h}$ of $\phi(N A, h(N)) /(N l(A))$ is equal to $\nu_{\tilde{\theta}_{0}} / \cos \left(\widetilde{\theta}_{0}-\theta\right)$ for some $\widetilde{\theta}_{0}$. Thus we divide $\operatorname{cyl}(N A, h(N))$ into slabs orthogonal to $\vec{v}\left(\widetilde{\theta}_{0}\right)$ instead of slabs orthogonal to $\vec{v}(\theta)$. Thus we compare $\phi(N A, h(N))$ with $h(N)$ sums of $N l(A) / \cos \left(\widetilde{\theta}_{0}-\theta\right)$ terms equal in law with $\tau_{n}\left(\widetilde{\theta}_{0}\right)$, the maximal flow from the upper half part to the lower half part of the boundary of a box of size $n \times h(n)$ oriented towards the direction $\widetilde{\theta}_{0}$. We conclude again thanks to Cramér's Theorem in $\mathbb{R}$.

If (F4) is not satisfied, we cannot use Cramer's Theorem. However, we can perform the division of $\operatorname{cyl}(N A, h(N))$ into slabs orthogonal to $\widetilde{\theta}_{0}$, and thus obtain an equation very close to (4.34):

$$
\mathbb{P}[\phi(N A, h(N)) \geq \lambda N l(A)] " \leq " \mathbb{P}\left[\tau_{N, n}^{i} \geq \lambda N l(A)\right]^{p h(N)},
$$

where $\tau_{N, n}^{i}$ is the maximal flow from the upper half part to the lower half part of the boundary of a slab. Thus, if (H3) holds instead of (F4), equation (4.35) leads to the conclusion of Lemma 4.6.

Remark 4.8. The hypotheses (H3) or (F4) may not be optimal, but a simple example shows why we need such kind of hypotheses. We consider that the capacity of an edge is distributed according to the Pareto law of parameters $p$ and 1 , i.e., the probability that an edge has a capacity bigger than $t \geq 1$ is equal to $t^{-p}$. We consider the rectangle $A=[0,1] \times\{0\}$, and the maximal flow $\phi(n A, h(n))$ from the top to the bottom of the cylinder $[0, n] \times[-h(n), h(n)]$. If all the vertical edges $\left(e_{i}, i=1, \ldots, 2 h(n)\right)$ (we suppose $h(n) \in \mathbb{N}$ for simplicity) in the box that are included in the segment $\{1\} \times[-h(n), h(n)]$ have a capacity bigger than $\lambda n$ for a fixed $\lambda$, then $\phi(n A, h(n))$ is bigger than $\lambda n$. We obtain:

$$
\mathbb{P}[\phi(n A, h(n)) \geq \lambda n] \geq \mathbb{P}\left[\forall i=1, \ldots, 2 h(n), t\left(e_{i}\right) \geq \lambda n\right] \geq(\lambda n)^{-2 p h(n)} .
$$

If $h(n) \log n$ is not large compared to $n$, in the sense that $h(n) \log n / n$ does not converge towards $+\infty$, then equation (4.33) is not satisfied.

\subsubsection{End of the proof of Theorem 2.9}

For this last section, we impose $($ H1 $),($ H2) $,($ F1 $),($ F2), $($ FH1 $),($ FH2) and either $(\mathbf{F} 4)$ or $($ H3). Let $\mathcal{F}$ be a closed subset of $\mathbb{R}^{+}$. We want to prove that

$$
\limsup _{n \rightarrow \infty} \frac{1}{n l(A)} \log \mathbb{P}\left[\frac{\phi_{n}}{n l(A)} \in \mathcal{F}\right] \leq-\inf _{\lambda \in \mathcal{F}} \mathcal{K}_{\theta, h}(\lambda) .
$$


If $\eta_{\theta, h}$ belongs to $\mathcal{F}$, then according to Corollary 2.5 , we know that

$$
\lim _{n \rightarrow \infty} \mathbb{P}\left[\frac{\phi_{n}}{n l(A)} \in \mathcal{F}\right]=1
$$

and so

$$
\limsup _{n \rightarrow \infty} \frac{1}{n l(A)} \log \mathbb{P}\left[\frac{\phi_{n}}{n l(A)} \in \mathcal{F}\right]=0=-\inf _{\lambda \in \mathcal{F}} \mathcal{K}_{\theta, h}(\lambda)
$$

because $\mathcal{K}_{\theta, h}$ is non-negative, and $\mathcal{K}_{\theta, h}\left(\eta_{\theta, h}\right)=0$. Let us suppose that $\eta_{\theta, h}$ does not belong to $\mathcal{F}$. The following proof is similar to the one of the upper bound in Rossignol and Théret [14]. We define $f_{1}=\sup \left(\mathcal{F} \cap\left[0, \eta_{\theta, h}\right]\right)$ and $f_{2}=\left(\inf \mathcal{F} \cap\left[\eta_{\theta, h},+\infty[)\right.\right.$. We suppose here that $\mathcal{F} \cap\left[0, \eta_{\theta, h}\right]$ and $\mathcal{F} \cap\left[\eta_{\theta, h},+\infty[\right.$ are non empty, because it is the most complicated case (if one of these two sets is empty, part of the following study is sufficient). Since $\mathcal{F}$ is closed, we know that $f_{1}<\eta_{\theta, h}$ and $f_{2}>\eta_{\theta, h}$. Then

$$
\limsup _{n \rightarrow \infty} \frac{1}{n l(A)} \log \mathbb{P}\left[\frac{\phi_{n}}{n l(A)} \in \mathcal{F}\right] \leq \limsup _{n \rightarrow \infty} \frac{1}{n l(A)} \log \left(\mathbb{P}\left[\frac{\phi_{n}}{n l(A)} \leq f_{1}\right]+\mathbb{P}\left[\frac{\phi_{n}}{n l(A)} \geq f_{2}\right]\right),
$$

On one hand, by (3.18), we know that

$$
\limsup _{n \rightarrow \infty} \frac{1}{n l(A)} \log \mathbb{P}\left[\phi_{n} \leq f_{1} n l(A)\right] \leq-\mathcal{K}_{\theta, h}\left(f_{1}\right)
$$

On the other hand, if we refer to Lemma 4.6, we know that

$$
\limsup _{n \rightarrow \infty} \frac{1}{n l(A)} \log \mathbb{P}\left[\phi_{n} \geq f_{2} n l(A)\right]=-\infty .
$$

If $\mathcal{K}_{\theta, h}\left(f_{1}\right)=+\infty$, we have

$$
\limsup _{n \rightarrow \infty} \frac{1}{n l(A)} \log \mathbb{P}\left[\frac{\phi_{n}}{n l(A)} \in \mathcal{F}\right]=-\infty=-\inf _{\mathcal{F}} \mathcal{K}_{\theta, h},
$$

because $\mathcal{K}_{\theta, h}$ is infinite on $\left[0, f_{1}\right]\left(\mathcal{K}_{\theta, h}^{\prime}\right.$ is non-increasing $)$ and on $\left[f_{2},+\infty\left[\right.\right.$, so on $\mathcal{F}$. If $\mathcal{K}_{\theta, h}\left(f_{1}\right)<\infty$, we have

$$
\limsup _{n \rightarrow \infty} \frac{1}{n l(A)} \log \mathbb{P}\left[\frac{\phi_{n}}{n l(A)} \in \mathcal{F}\right] \leq-\mathcal{K}_{\theta, h}\left(f_{1}\right)=-\inf _{\mathcal{F}} \mathcal{K}_{\theta, h}
$$

because $\mathcal{K}_{\theta, h}$ is non-increasing on $\left[0, f_{1}\right]$ and infinite on $\left[f_{2},+\infty[\right.$. So the upper bound is proved.

\section{REFERENCES}

[1] D. Boivin, Ergodic theorems for surfaces with minimal random weights. Ann. Inst. Henri Poincaré Probab. Stat. 34 (1998) 567-599.

[2] B. Bollobás, Graph theory. An introductory course, edited by Springer-Verlag, New York. Graduate Texts in Mathematics 63 (1979).

[3] R. Cerf, The Wulff crystal in Ising and percolation models, in École d'Été de Probabilités de Saint Flour, edited by SpringerVerlag. Lect. Notes Math. 1878 (2006).

[4] R. Cerf and M. Théret, Law of large numbers for the maximal flow through a domain of $\mathbb{R}^{d}$ in first passage percolation. Trans. Amer. Math. Soc. 363 (2011) 3665-3702.

[5] R. Cerf and M. Théret, Lower large deviations for the maximal flow through a domain of $\mathbb{R}^{d}$ in first passage percolation. Probab. Theory Relat. Fields 150 (2011) 635-661.

[6] R. Cerf and M. Théret, Upper large deviations for the maximal flow through a domain of $\mathbb{R}^{d}$ in first passage percolation. To appear in Ann. Appl. Probab., available from arxiv.org/abs/0907.5499 (2009c). 
[7] J. T. Chayes and L. Chayes Bulk transport properties and exponent inequalities for random resistor and flow networks. Commun. Math. Phys. 105 (1986) 133-152.

[8] O. Garet, Capacitive flows on a $2 d$ random net. Ann. Appl. Probab. 19 (2009) 641-660.

[9] G. Grimmett and H. Kesten, First-passage percolation, network flows and electrical resistances. Z. Wahrsch. Verw. Gebiete 66 (1984) 335-366.

[10] J.M. Hammersley and D.J.A. Welsh, First-passage percolation, subadditive processes, stochastic networks, and generalized renewal theory, in Proc. Internat. Res. Semin., Statist. Lab. Univ. California, Berkeley, Calif. Springer-Verlag, New York (1965) 61-110.

[11] H. Kesten, Aspects of first passage percolation, in École d'été de probabilités de Saint-Flour, XIV-1984, edited by Springer, Berlin. Lect. Notes Math. 1180 (1986) 125-264.

[12] H. Kesten, Surfaces with minimal random weights and maximal flows: a higher dimensional version of first-passage percolation. Illinois J. Math. 31 (1987) 99-166.

[13] R. Rossignol and M. Théret, Law of large numbers for the maximal flow through tilted cylinders in two-dimensional first passage percolation. Stoc. Proc. Appl. 120 (2010) 873-900.

[14] R. Rossignol and M. Théret, Lower large deviations and laws of large numbers for maximal flows through a box in first passage percolation. Ann. Inst. Henri Poincaré Probab. Stat. 46 (2010) 1093-1131.

[15] M. Théret, Upper large deviations for the maximal flow in first-passage percolation. Stoc. Proc. Appl. 117 (2007) 1208-1233.

[16] M. Théret, On the small maximal flows in first passage percolation. Ann. Fac. Sci. Toulouse 17 (2008) 207-219.

[17] M. Théret, Grandes déviations pour le flux maximal en percolation de premier passage. Ph.D. thesis, Université Paris Sud (2009a).

[18] M. Théret, Upper large deviations for maximal flows through a tilted cylinder. To appear in ESAIM: Probab. Stat., available from arxiv.org/abs/0907.0614 (2009b).

[19] Y. Zhang, Critical behavior for maximal flows on the cubic lattice. J. Stat. Phys. 98 (2000) 799-811.

[20] Y. Zhang, Limit theorems for maximum flows on a lattice. Available from arxiv.org/abs/0710.4589 (2007). 